University of Massachusetts Amherst

ScholarWorks@UMass Amherst

Masters Theses

Dissertations and Theses

October 2018

\title{
Evaluation and Validation of Distraction Detection Algorithms on Multiple Data Sources
}

Shashank Mehrotra

University of Massachusetts Amherst

Follow this and additional works at: https://scholarworks.umass.edu/masters_theses_2

Part of the Ergonomics Commons, and the Industrial Engineering Commons

\section{Recommended Citation}

Mehrotra, Shashank, "Evaluation and Validation of Distraction Detection Algorithms on Multiple Data Sources" (2018). Masters Theses. 710.

https://doi.org/10.7275/12668920 https://scholarworks.umass.edu/masters_theses_2/710

This Open Access Thesis is brought to you for free and open access by the Dissertations and Theses at ScholarWorks@UMass Amherst. It has been accepted for inclusion in Masters Theses by an authorized administrator of ScholarWorks@UMass Amherst. For more information, please contact scholarworks@library.umass.edu. 


\title{
EVALUATION AND VALIDATION OF DISTRACTION DETECTION ALGORITHMS ON MULTIPLE DATA SOURCES
}

\author{
A Thesis Presented
}

By

SHASHANK KUMAR MEHROTRA

Submitted to the Graduate School of the

University of Massachusetts Amherst in partial fulfilment

of the requirements for the degree of

MASTER OF SCIENCE IN INDUSTRIAL ENGINEERING \& OPERATIONS RESEARCH

September 2018

Mechanical \& Industrial Engineering 


\title{
EVALUATION AND VALIDATION OF DISTRACTION DETECTION ALGORITHMS ON MULTIPLE DATA SOURCES
}

\author{
A Thesis Presented
}

By

\section{SHASHANK KUMAR MEHROTRA}

Approved as to style and content by:

Shannon C Roberts Ph.D., Chair

Jenna L. Marquard Ph.D., Member

Michael Knodler Jr., Ph.D., Member

Sundar Krishnamurty, Department Head

Mechanical \& Industrial Engineering 


\section{ACKNOWLEDGEMENTS}

First, I would like to thank my advisor- Dr. Roberts for her continuous guidance, support and help from the problem definition phase to the writing of this manuscript. Her support has been one of the motivating factors for this study and without her help, this would not have been possible.

I thank all the members on my committee who gave me relevant and timely feedback. Their invaluable suggestions helped me think about focusing on the relevant aspects of my research.

I thank my research group member and friend- Fangda Zhang for his help towards the simulator study. The design for all the scenarios was done by him. I am grateful for his support and look forward to working with him in the future.

I would like to thank the Human Performance Lab for conducting the simulator study. I also thank all the participants who took time to participate in this study, which is the basis for all the simulator data.

Lastly, I would like to thank my parents and friends for supporting me in my decision to pursue research. 


\begin{abstract}
EVALUATION AND VALIDATION DISTRACTION DETECTION ALGORITHMS ON

MULTIPLE DATA SOURCES
\end{abstract}

SEPTEMBER 2018

SHASHANK KUMAR MEHROTRA

B.E., PES INSTITUTE OF TECHNOLOGY, BANGALORE

M.S. I.E.O.R., UNIVERSITY OF MASSACHUSETTS AMHERST

Directed by Professor Shannon C. Roberts

This study aims to evaluate algorithms designed to detect distracted driving. This includes the comparison of how efficiently they detect the state of distraction and likelihood of a crash. Four algorithms that utilize measures of cumulative glance, past glance behavior, and glance eccentricity were used to understand the distracted state of the driver and were validated on two separate data sources (i.e., simulator and naturalistic data). Additionally, an independent method for distraction detection was designed using data mining methods. This approach utilized measures like steering degree, lane offset, lateral and longitudinal velocity, and acceleration. The results showed a higher likelihood of distracted events when cumulative glances were considered. However, the state of distraction was observed to be higher when glance eccentricity was added. Additionally, it was observed that glance behavior using the four legacy algorithms were better detectors of the state of distraction as compared to the data mining method that used vehicular measures. This research has implications in understanding the state of distraction, predicting the 
power of different methods, and comparing approaches in different contexts (naturalistic vs simulator). These findings provide the fundamental building blocks towards designing advanced mitigation systems that give drivers feedback in instances of high crash likelihood. 


\section{CONTENTS}

Page

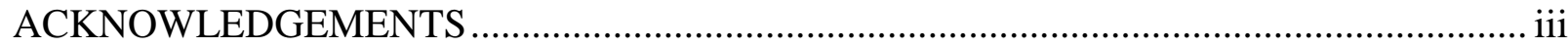

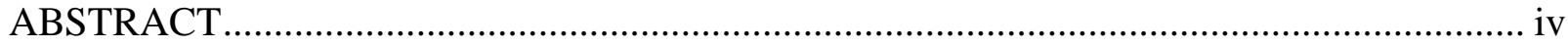

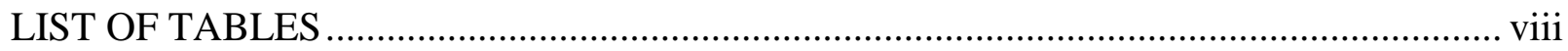

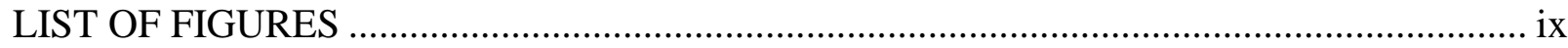

CHAPTER

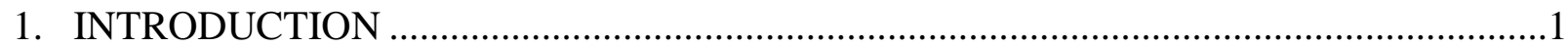

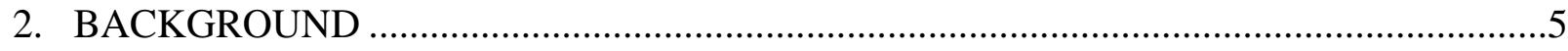

2.1 Definition of distraction ......................................................................................

2.1.1 Driver Restricted Attention (DRA) ..................................................

2.1.2 Driver Misprioritized Attention (DMPA) ............................................

2.1.3 Driver Neglected Attention (DNA) .......................................................

2.1.4 Driver Cursory Attention (DCA) ….....................................................

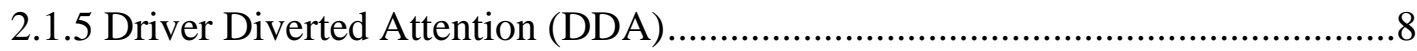

2.2 Attention, information processing, and decision-making models ............................... 9

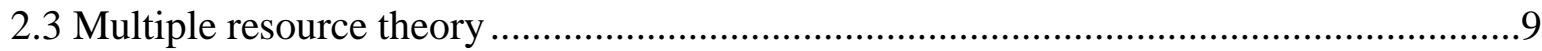

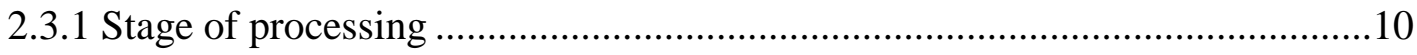

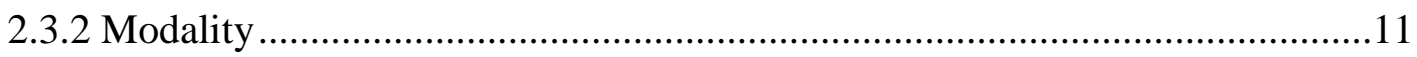

2.3.3 Access and response (code of processing) .............................................. 11

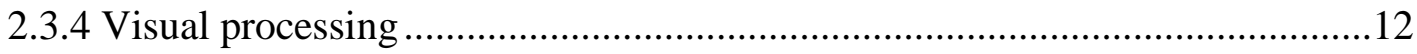

2.4 Attention selection and multitasking in driving .............................................. 12

2.4.1 Information processing models ..........................................................13

2.4.2 Decision making models ............................................................. 14

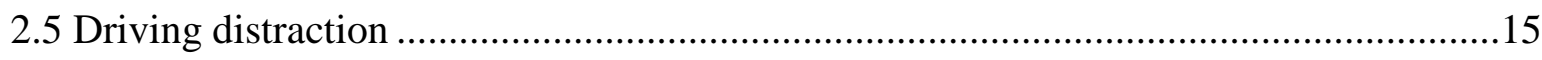

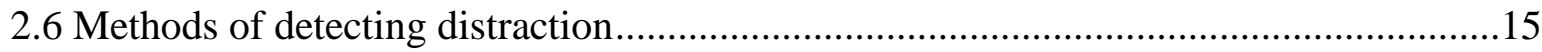

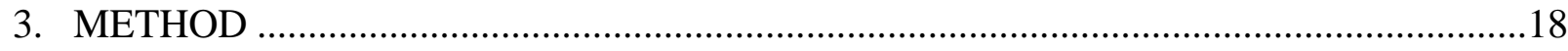


3.1 Legacy algorithms ..........................................................................................18

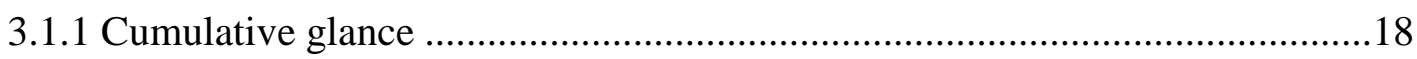

3.1.2 Cumulative glance current duration .............................................................19

3.1.3 Cumulative glance current duration \& glance eccentricity................................20

3.1.4 Buffered-cumulative glance current duration \& glance eccentricity ...................21

3.2 Inputs and outputs for each legacy algorithm ..............................................................24

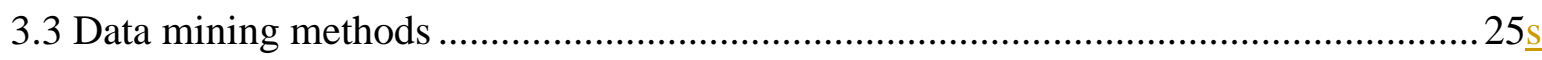

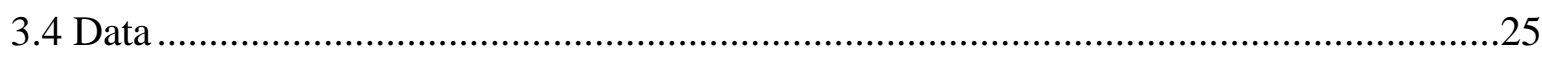

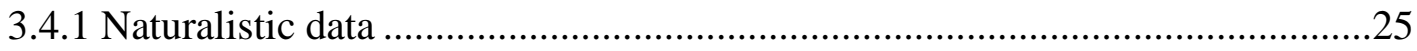

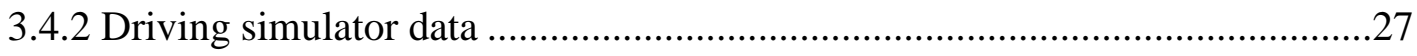

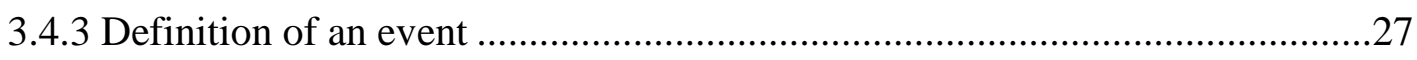

3.5 Experimental Design..............................................................................................27

3.5.1 Recruitment .........................................................................................2

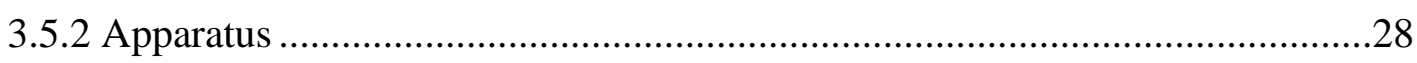

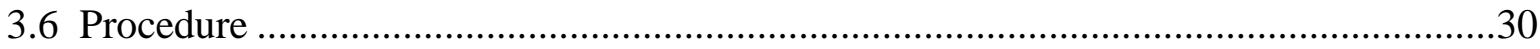

3.7 Distracting tasks ................................................................................................... 31

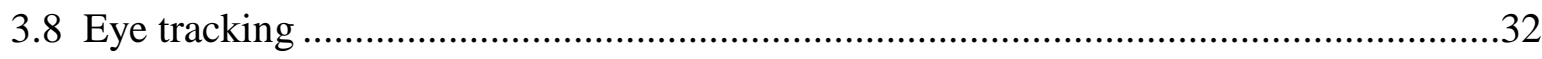

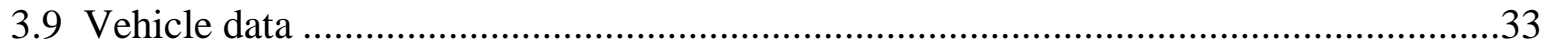

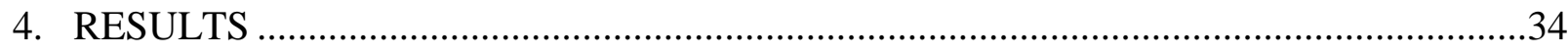

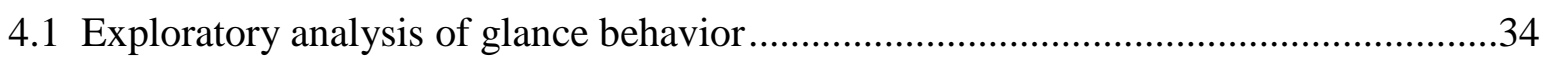

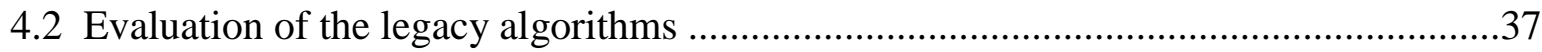

4.3 Impact of distraction on vehicle measures .....................................................................39

4.4 Detection of distraction using support vector machines ..................................................39

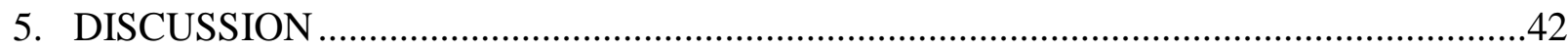

5.1 Detection of distraction and events due to the legacy algorithms ................................42

5.2 Vehicle data in detecting distraction ......................................................................

5.3 Limitations and future work...................................................................................44

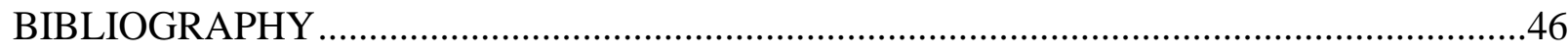




\section{LIST OF TABLES}

Table

Page

Table 1: Definition of the ellipses for each glance location in the CGCDGE algorithm 21

Table 2: Classification of each glance location for the Buffered-CGCDGE algorithm 22

Table 3: Penalization for glance transitions for the Buffered-CGCDGE algorithm.................. 23

Table 4: Input and Output for each legacy algorithm 24

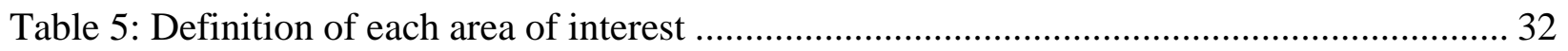

Table 6: Correlation matrix for vehicle measures............................................................... 40

Table 7: Distraction detection results for machine learning methods..... 


\section{LIST OF FIGURES}

Figure

Page

Figure 1: Taxonomy of driver inattention (Regan et al., 2011) 7

Figure 2: A 3D representation of the stages, modalities, codes, and visual processing based on MRT (Wickens, 2008) ........ 11

Figure 3: A model of Human Information Processing (Wickens et al.,2015)..... 14

Figure 4: The hierarchy structure of the road task (Michon, 1985)..... 15

Figure 5: The driving simulator 29

Figure 6: The eye tracker used to record glance behavior 30

Figure 7: Visual distraction task 31

Figure 8: Combined distraction task 32

Figure 9: Glance locations (excluding Forward) for the distracted participants 5 seconds before the crash in the 100 Cars dataset.

Figure 10: Glance locations (excluding Forward) for the distracted participants 5 seconds before the crash for the simulator data 36

Figure 11: Odds ratios for detecting distraction for different time windows 38

Figure 12: Odds ratios for detecting an event for different time windows 38 


\section{CHAPTER 1}

\section{INTRODUCTION}

Distracted driving has been long recognized as an important epidemiological issue in the United States with major loss of life and property reported each year. According to the National Highway Transportation and Safety Administration (NHTSA), out of the 37,461 crash related fatalities in 2016, 3,450 fatalities were attributed to distracted driving (NHTSA, 2017). In terms of economic damage, distracted driving crashes caused $\$ 40$ billion in economic costs and were responsible for $16 \%$ of all economic impacts from motor vehicle crashes in 2010 (Kahn \& Gotschall, 2015).

Several policy changes and measures have been taken to prevent drivers from distracted driving behavior (Vegega, Jones, \& Monk, 2013) and the number of distracted driving fatalities has declined. Despite growing awareness about distraction, eliminating injuries and fatalities due to distracted driving poses a major challenge for the research community and policy makers alike. The National Safety Council (NSC) reported in 2015 that nearly 27\% of all crashes involved texting. However, NSC reports that there is a huge under-reporting of such crashes, which leads to a lack of understanding of the severity of the issue (National Safety Council, 2013). Apart from the use of technology while driving, distraction has also been reported due to influences of passengers (Caird, Simmons, Wiley, Johnston, \& Horrey, 2018) and drowsiness (Yeo, Li, Shen, \& Wilder-Smith, 2009) (MacLean, Davies, \& Thiele, 2003).

There are two main types of distractions based on the task the driver is performing. Tasks that cause eyes-off-the-road are commonly known as visual distractions since they divert focal vision from the road and diminish the driver's ability to anticipate hazards. This causes 
degradation in lane keeping, even though drivers can perceive the outline of the roadway (Horrey $\&$ Wickens, 2004). Likewise, tasks that take the driver's mind-off-the-road are termed cognitive distractions. It reflects the shared central processing demand of driving and secondary tasks. Cognitive distraction does not directly interfere with focal vision and is therefore less disruptive to hazard detection and vehicle control compared to visual distraction. Liang et al., (2009) explored the effects of combined distractions (audio-visual) on driving performance. It was found that frequent off-road glances, longer total eye-off-road time, and high gaze concentration in cases when drivers look at the road tend to cause high deviation in lane positioning and discrete steering correction. Apart from visual and cognitive distractions, manual distraction also plays a role in influencing driving performance. Manual distraction involves the performance of a task by using one's hands or feet, like eating or drinking. For the purposes of this research, manual distraction was not considered.

Researchers over the past decade have established several frameworks that have established how distraction leads to impairment. Distracted driving finds resonance in the multiple resource theory (MRT) proposed by Wickens (2002). MRT explains how multiple tasks influence performance, the interference of tasks performed concurrently, and its consequences on performance predictions. Additionally, Engstrom et al., (2013) proposed how biases in perception of sensory inputs, values, contexts, and cognition affect the outcome of decisions. Additionally, several frameworks have been proposed by Shinar et al., (2017) to explain the influence of experience on driving performance and how strategic levels of maneuvering while driving would influence their performance. A majority of past research considers these frameworks as the guiding principle upon which the effect of distraction is measured and helps 
classify the impact different types of distractions have on driving (Liang, Lee, \& Yekhshatyan, 2012).

With a growing understanding of how distraction impacts driving, there has been an emphasis on understanding methods to determine distraction and model them algorithmically. Work done by Senders et al., (1967) helped model the relationship between vehicle parameters and driving uncertainty. Additionally, eye-glance behavior has been extensively explored to understand how it impacts the degree of distraction. Several visual distraction detection algorithms have been developed (Donmez et al., 2007; J Engström \& Mårdh, 2007; Fletcher \& Zelinsky, 2007; Kircher \& Ahlström, 2009; Klauer et al., 2010; Pohl et al., 2007) that can help determine the distracted state of the driver. These approaches are based on evaluating the glance patterns and using deterministic methods to classify the state of the driver.

While traditional approaches have been developed, over the last few years, a wide range of data mining methods have been applied to determine the state of distraction. Work done by Schwarz et al. (2016) looked to establish a methodology using a random forest algorithm along with vehicle and driver-based data. Research done by Lee et al. (2013) explored relationships between distraction and driving performance decrements by exploring vision-based algorithms around eyes off the road. Liang et al. (2007) explored the use of Support vector machines (SVM) in predicting the likelihood of crashes attributed to visual distraction. Additionally, data mining approaches have been useful in drowsiness detection by Mc Donald et al. (2013) and Yeo et al. (2009).

When exploring different methods to detect distraction, it is important to consider the type of data that is used. More specifically, it is important to consider whether the data was 
generated in a naturalistic study or was obtained from a controlled experimental environment. Liang et al., (2012) explored the effect of visual distraction in the case of 100 cars naturalistic driving study. Additionally, SHRP2 has been used by Victor et al. (2015) to understand the relationship between glance behavior, driver inattention, and crash risk across different dimensions like the risk of precipitation events. In comparison, several studies have been done in a simulated environment to understand the influence of distraction. Studies by Donmez et al. (2008), Schwarz et al. (2016), and Liang et al. (Liang et al., 2007) (2010) are focused on understanding visual and cognitive distractions based on data obtained from driving simulators.

While past research has explored the nature of driver distraction through different methods, there a few drawbacks of these algorithms. While almost all of these have been developed based on naturalistic data or simulator data, there has been no work to cross validate the results on different data sources. Also, while most of the algorithms either depend on the eye glance data or vehicular parameters, there has been little research on exploring how more robust methods can be established by combining these data.

This study aims to:

1. validate distraction detection algorithms (legacy algorithms) based on the glance location, duration, and eccentricity across two data sources.

2. explore how data mining methods compare to traditional methods in terms of detecting distraction and crash/near crash likelihood. 


\section{CHAPTER 2}

\section{BACKGROUND}

Driving related fatalities are a major public health issue in the United States. According to the Traffic Safety Facts 2016 (NHTSA, 2017), 37,461 people were killed due to the traffic related accidents. Apart from the fatalities, the economic cost is a major concern with $\$ 242$ billion lost in 2010 (Kahn \& Gotschall, 2015). Additionally, the indirect economic costs could inflate the costs to $\$ 836$ billion, which amounts to about $4.5 \%$ of the GDP of the United States. The figures paint a grim scenario of the total loss incurred due to motor vehicle crashes. This issue poses a huge challenge for human factors and transportation safety researchers who are search for methods to decrease these fatalities.

While several causes contribute to these fatalities, human choices that lead to crashes are an important area of focus. Human choices include speeding, alcohol-impairment, unrestrained passenger occupants, drowsiness, as well as distracted driving. Estimates report that $94 \%$ of the crashes can be attributed to human error (Singh, 2015). Additionally, Lee (2008) stated that in the past 50 years of research in transportation safety, the continuous challenge to reducing traffic fatalities is improving driver behavior. A few programs like Mothers Against Drunk Driving (Fell \& Voas, 2006) have been successful in reducing the increase of alcohol related crashes. Past research done by Shults et al. (2004) establishes the effectiveness of laws against unrestrained occupants while driving. These studies establish how increased awareness and policy changes can enforce safer choices while driving.

One specific human choice that causes traffic fatalities is distracted driving. While several states have imposed strict laws about texting while driving (Goodwin, O’Brien, \& Foss, 2012; 
Ibrahim, Anderson, Burris, \& Wagenaar, 2011), the laws have not resulted in sustained positive effects. While there were fewer fatalities due to distracted driving in 2016 as compared to 2015, the NSC (National Safety Council, 2013) states under reporting of distracted driving caused by cell phones. Additionally, with the advent of technologically advanced in vehicle information systems (IVIS), the use of such systems while driving can cause interference between the primary driving task and the secondary task (Kim, Kwon, Heo, Lee, \& Chung, 2014)(Young, 2012).

In the remainder of this Background section, distraction is explored in the context of driving. First, this section looks to define distraction and various aspects of how distraction impacts driving. Second, a theoretical foundation of distraction from the point of view of attention allocation and the multiple resource theory is explored. Additionally, methods of detecting driving distraction and how they are useful in categorizing distraction are covered. Finally, the algorithms for detecting distractions and crash like events are explored.

\subsection{Definition of distraction}

Regan et al., (2011) explained the umbrella term Driver Inattention as a phenomenon "when the driver's mind has wandered from the driving task for some non-compelling reason" such as when the driver is "focusing on internal thoughts (i.e., daydreaming, problem solving, worrying about family problems, etc.) and not focusing attention on the driving task". Inattention is defined both by driver activity (for example, driver speaking on the phone while driving) and driver state (for example, drowsiness). This would lead us to categorize different aspects of driver inattention based on the activity and state of the driver. Figure 1 shows the categorization of different types of distraction. 


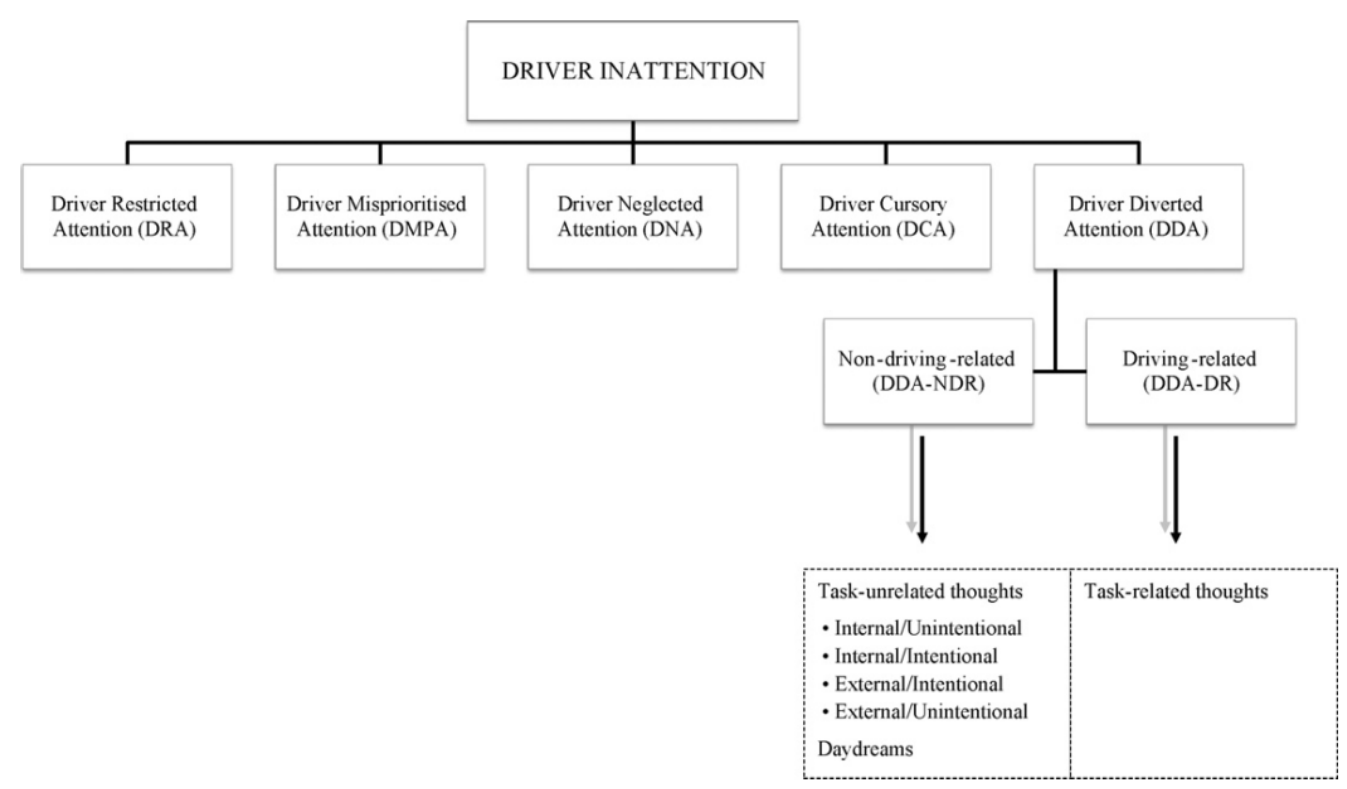

Figure 1: Taxonomy of driver inattention (Regan et al., 2011)

\subsubsection{Driver Restricted Attention (DRA)}

Lack of attention to critical activities for ensuring that safe driving happens, which is linked to something that physically prevents (due to biological factors) the driver from detecting (and hence from attending to) information critical for safe driving.

\subsubsection{Driver Misprioritized Attention (DMPA)}

Lack of attention to critical activities for ensuring that safe driving happens. Hancock et al., (2009) explained DMPA as "engaged in what is considered 'the wrong' aspect of the driving task at the time in question".

\subsubsection{Driver Neglected Attention (DNA)}


Lack of attention to activities critical for safe driving brought about by the driver neglecting to attend to activities critical for safe driving. For example, a driver approaching an unsignalized T- intersection intending to turn right who does not see a cyclist on a parallel bike path approaching from the right because the driver does not look in this direction, as defined by Engstrom et al. (2009).

While DNA and DMPA appear similar, the priority in DMPA is in safely driving while focusing on a single aspect of driving. DNA is a lack of safe driving altogether.

\subsubsection{Driver Cursory Attention (DCA)}

Insufficient or no attention to activities critical for safe driving brought about by the driver giving cursory or hurried attention to activities critical for safe driving. An example is a driver who is in a hurry and does not look both ways when merging and, in doing so, ends up colliding with another vehicle.

\subsubsection{Driver Diverted Attention (DDA)}

The diversion of attention away from activities critical for safe driving toward a competing activity, which may result in insufficient or no attention to activities critical for safe driving. This category of inattention is synonymous with "driver distraction". The definition proposed here is almost identical to that coined for driver distraction by Lee et al. (2013): "Driver distraction is a diversion of attention away from activities critical for safe driving toward a competing activity." This is the base definition that used to define distraction. 
Werneke et al., (2012) states that the causes for distraction related errors were due to one of the following:

1. drivers looked but failed to see, or

2. drivers failed to look, and thus saw the other traffic participant too late to avoid a collision.

Keeping this in mind, it becomes essential to study how drivers make decisions while inattentive. To understand this decision-making process, it is important to establish how drivers maintain attention and process information based on their expertise. In the next section, these viewpoints are explained in detail.

2.2 Attention, information processing, and decision-making models

To understand attention allocation, it is important to explore the theoretical foundations of attention as well as how attention impacts information processing and driver decision making. This section introduces the theory behind inattention. First, the multiple resource theory is explored because it serves as the theoretical foundation of this research. Then, focus on attention selection in the context of driving is explained. Further, this section explores how task selection and prioritization is done in the context of driving.

2.3 Multiple resource theory

Multiple Resource Theory (MRT) is a theory of understanding performance when a person wants to perform multiple tasks. The implications of this theory stem from the predictions regarding the ability of a driver to perform while multi-tasking, which causes a higher workload. 
Theoretically, this theory helps explain and predict the dual task interference between concurrently performed tasks.

The multiple resource theory proposes that there are four important categorical and dichotomous dimensions that account for variance in time-sharing performance. The dimensions are: (1) stage of processing (2) modalities involved in perceiving the task (3) codes (access and responses) and (4) visual processing. Each of these dimensions has two discrete 'levels'. The theory assumes that two tasks that demand the same level of a given dimension (e.g., two tasks demanding visual perception) will interfere with each other more than two tasks that demand different levels on the given dimension (e.g. one visual and one auditory task). Figure 2 represents these four dimensions in greater detail.

\subsubsection{Stage of processing}

The resources used for perceptual/cognitive activities, e.g., involving working memory, appear to be the same, and those resources are functionally separate from those underlying the selection and execution of responses. Evidence for this dichotomy is provided when the difficulty of responding in a task is varied but does not affect performance of a concurrent task whose demands are more perceptual and cognitive in nature. Conversely, another example is when increases in perceptual cognitive difficulty do not influence the performance of a concurrent task whose demands are primarily response-related. 


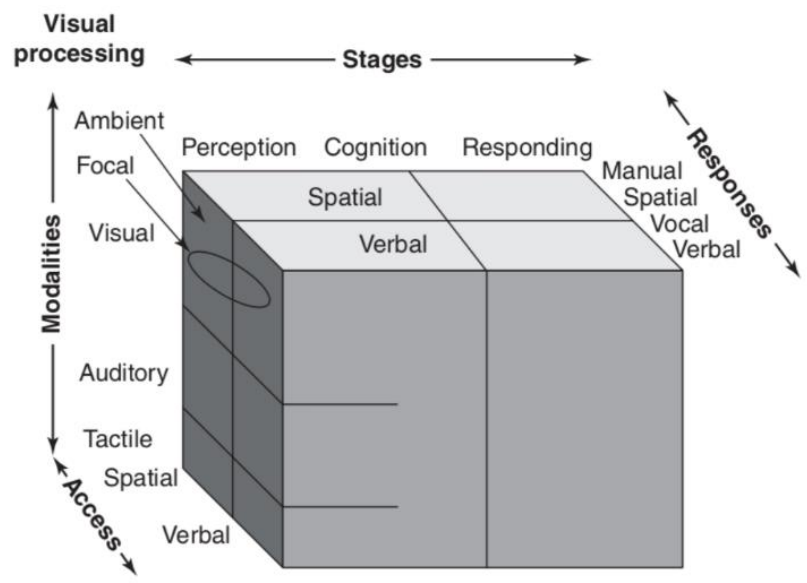

Figure 2: A 3D representation of the stages, modalities, codes, and visual processing based on MRT (Wickens, 2008)

\subsubsection{Modality}

Division for attention between the visual and auditory channels is better than between two auditory channels or two visual channels. This implies that cross-modal time-sharing is better than intra-modal time-sharing. Two competing visual channels, if they are far enough apart, will require visual scanning between them, at an added cost. If they are too close together, they may impose confusion and masking, just as two auditory messages may mask one another if they occupy nearby or overlapping temporal frequencies.

\subsubsection{Access and response (code of processing)}

This dimension defines the distinction between analogue and verbal processes. This accounts for the relatively high degree of efficiency with which manual and vocal responses can be time-shared, if manual responses are usually spatial in nature (e.g., tracking, steering, joystick, or mouse movement) and vocal ones are usually verbal in nature (e.g., speaking). 


\subsubsection{Visual processing}

This dimension helps differ between focal vision and ambient vision. Focal vision is primarily (but not exclusively) foveal and supports object recognition and high acuity perception, such as that involved in reading text and recognizing symbols (Wickens, 2008). The distribution of the ambient vision is across the entire visual field and preserves its competency in peripheral vision.

\subsection{Attention selection and multitasking in driving}

Engstrom et al. (2013) suggested that the process of selection of attention involves the selection of a schemata which is set to be activated, with attention being the outcome of this process. The two types of schemata are defined as: a) basic (i.e., sensory motor and semanticvision for action and vision for perception) and b) task context (i.e., functional representation in parallel). The selection of schema are based either on habitual selection due to associated task context or deliberate selection due to cognitive control. Actions driven by cognitive control may be viewed as "willed" or self-regulated as opposed to those triggered by the schema system alone. Cognitive control is required in cases whenever there is a conflict in schema selection that cannot be resolved by routine, automatic scheduling alone. The selection of schema is based on the interactive flow of facilitations and inhibitions, which may cause the schema with the strongest activation to prevail while the competing schema is suppressed or fails. Lastly, the process of selecting the schema is based on the value system, which is determined by the relevance of stimuli and actions for behavioral goals. 


\subsubsection{Information processing models}

The information processing model is useful in understanding the sequence involved in the execution of the driving task. The information processing model contains three elements of cognition: perception, thinking and understanding of the world around the driver, as shown in Figure 3. The driver's sensory system gathers information, which is then perceived, and provides a meaningful interpretation, which is aided by prior knowledge. Perception leads directly to the selection and execution of a response, as when a driver swerves to avoid a converging car. However, some action is delayed, or not executed at all, as information in working memory is manipulated. Long-term memory is used to create a more permanent representation of the information. Central to all these processing is the dependency on the availability of the mental or cognitive (attention) resources. All of these processes are linked together with the feedback loop. The actions generate new information to be sensed and perceived. 


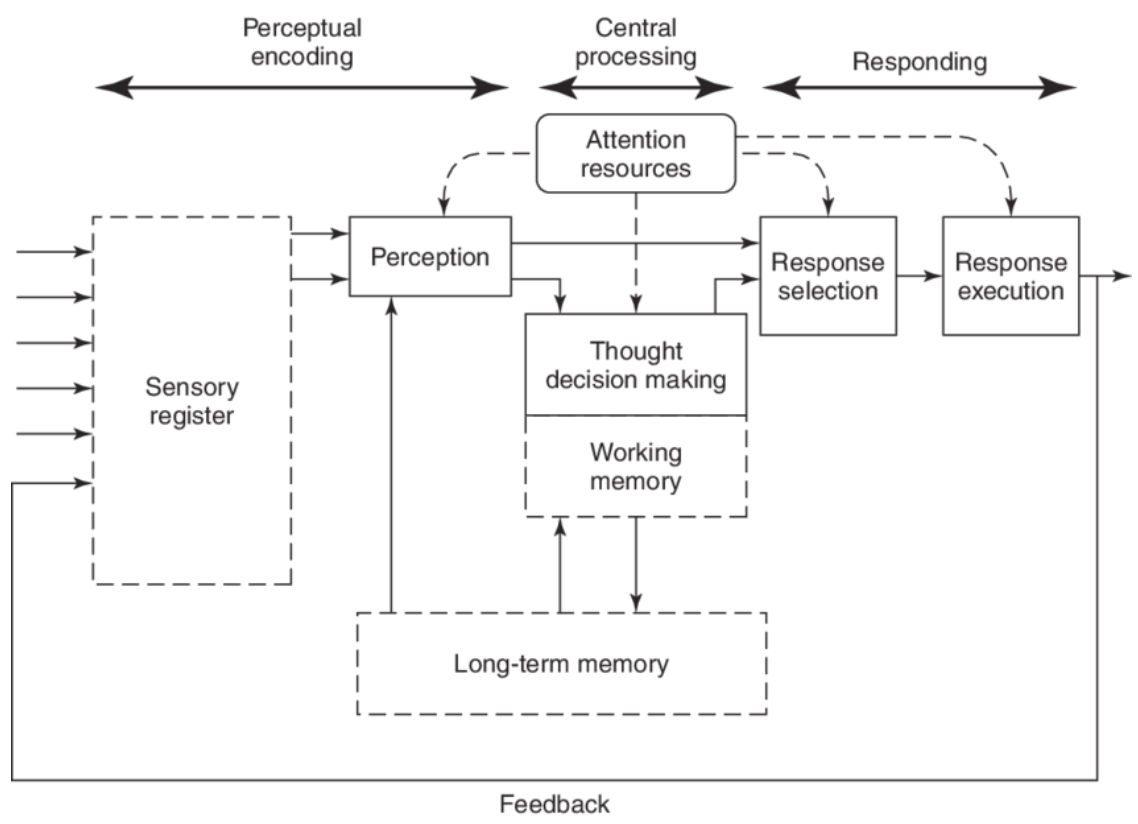

Figure 3: A model of Human Information Processing (Wickens et al., 2015)

\subsubsection{Decision making models}

Yet another model discusses how the driver makes decisions based on factors like how the trip is planned and when to brake. The model uses three levels of activity to describe the complex tasks that comprise driving: strategic, tactical, and control (Michon, 1985). Strategic tasks focus on the purpose of the trip and the drivers' overall goal, many of which are decided before the driver starts driving. For example, a strategic decision is to decide how to get to a location on a Monday morning. Tactical tasks focus on the choice of maneuvers and immediate goals in getting to a destination. This may include speed selection or headway maintenance. Finally, control tasks are focused on the momentary operation of the vehicle, such as maintaining the regulated speed and lane position. The hierarchy is described in Figure 4. 


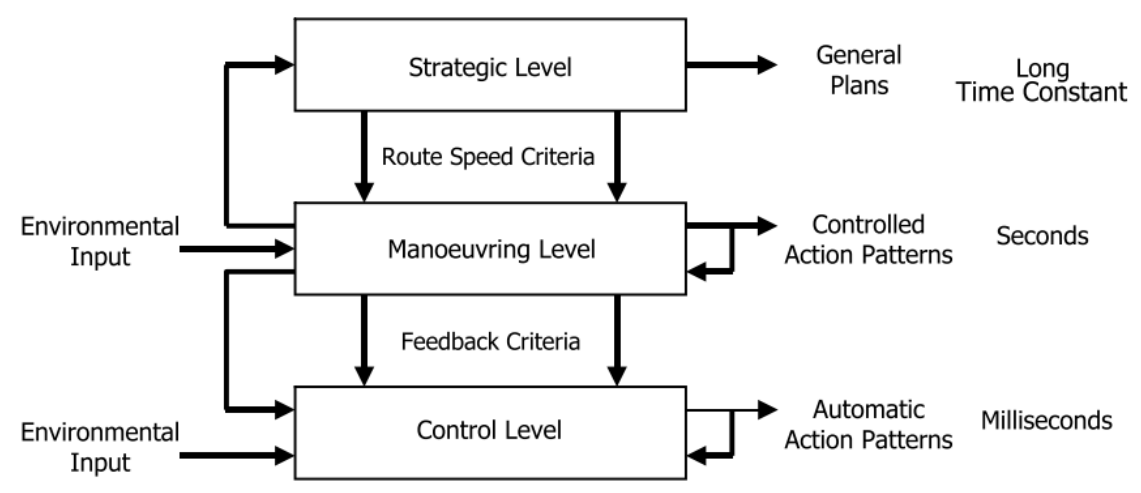

Figure 4: The hierarchy structure of the road task (Michon, 1985)

\subsection{Driving distraction}

This research considers MRT as the model to explain driving distraction as it best explains how multi-tasking affects performance based on the availability of resources and the structure of the task. In the context of MRT, to understand how the lack of working memory and increased demand cause driving inefficiency, it is important to contextualize these demands in the context of driving. Driving distractions reflect the shared central processing demand of driving and secondary tasks, which is in line with the theory. While distractions are disruptive, it is important to understand whether it is due to an increase in demand, the way the task is constructed or, if it affects one or more modalities. This research can potentially answer some of these questions through understanding distraction detection methods. In the next section, methods of distraction detection are introduced.

2.6 Methods of detecting distraction

In the context of distracted driving, a detection algorithm tries to determine the state of the driver based on their glance behavior or vehicle dynamics. These can provide a prediction of 
the likelihood of a crash like event. The detection criteria are established by determining whether a driver's glance behavior is in or around their field relevant for driving (FRD). The FRD, which includes the driver looking forward, at the speedometer, as well as looking at the left, right and rearview mirrors, are indicative of safe driving behavior. Any glance away from FRD is considered unsafe driving behavior and is penalized. For this research, algorithms using this criterion are referred to as "legacy" algorithms.

Four legacy algorithms were explored in this research. The first is the cumulative glance (CG) algorithm (Klauer, Dingus, Neale, Sudweeks, \& Ramsey, 2009) considers the total glances in a 6 second window when there is a crash or a near crash in the $5^{\text {th }}$ second of the window. The second is the cumulative glance current duration (CGCD) algorithm (Donmez, Boyle, Lee, \& Mcgehee, 2006), which considers the current glance or a glance that has occurred in the last 3 seconds. These glances are weighted, which gives an evaluation of the driver risk. Third, the cumulative glance current duration and glance eccentricity (CGCDGE) algorithm (Engstrom and Mardh, 2007) considers glance eccentricity and the summation of all the glances that occur before a crash like scenario. Finally, the Buffered cumulative glance and current duration (Buffer CGCD) algorithm (Kircher \& Ahlström, 2013) uses a buffer to track the progress of the driver. When the driver glances towards the speedometer and mirrors, the buffer is updated with a latency of $0.1 \mathrm{~s}$; any other glance is updated with a latency of $1 \mathrm{~s}$. All legacy algorithms work with the underlying principle that when drivers look away from the road, they will have poor driving performance and a higher likelihood of crashing.

While the above criteria are limited to FRD, it is not the only criteria and methodology used to determine the degree of distraction. Past research has established distraction detection estimates based on how drivers perform when subjected to distractions. A majority of this 
research has been done using machine learning methods. Work done by Schwartz (2016) uses random forest models based on vehicle and driver-based data. Research done by Lee et al., (2013) explored relationships between distraction and driving performance decrements by exploring vision-based methods using the criteria of eyes off the road. Liang et al., (2007) explored the use of support vector machines (SVM) in predicting the likelihood of crashes attributed to visual distraction.

In this research, both legacy as well as machine learning methods have been explored for detecting distraction and events. This analysis was conducted on two different data sources to understanding their validity. The next section focuses on the implementation and evaluation of these methods. 


\section{CHAPTER 3}

\section{METHOD}

In this research, legacy as well as machine learning methods have been explored for detecting distraction and events. The study is separated into two phases. The first phase of the analysis uses the glance behavior data in the naturalistic and simulator data. The objective in this phase is to understand the performance of the four legacy algorithms across data sources. The second phase involves the simulator data only. In this phase, distraction detection was explored using data mining methods, more specifically, Support Vector Machines (SVM).

This section explains the legacy algorithms and how they have been evaluated. Additionally, the section covers the experimental design, how the data was collected, and how the analysis was carried out. Finally, this section introduces SVMs as the method of classifying between different types of distractions.

\subsection{Legacy algorithms}

\subsubsection{Cumulative glance}

This legacy algorithm defines distraction as looking away from the road for two seconds within a six second window.

Step One:

Define the distracted locations versus the non-distracted locations; non-distracted locations are forward, rearview, right side mirror, left side mirror while the rest of the locations are classified as distracted. 
Step Two:

Identify whether the person is distracted or not distracted at each frame using the criteria explained in the first step.

Step Three:

For each driver, create a sliding six second window with the event occurring in the $5^{\text {th }}$ second. The number of distracted glances within the sliding window is then added. The legacy algorithm defines distraction (and a near-crash/crash event) as having a total of two distracted glances within the six second window. This operation is repeated for each driver.

\subsubsection{Cumulative glance current duration}

This legacy algorithm was primarily designed to measure distraction in terms of glances towards an in-vehicle information system (IVIS) display. For this research, there are no tasks that focus on the interaction with the IVIS. Thus, any glances away from the road were considered a distraction which include glances towards the passenger and looking at an object inside a car while driving.

Step One:

Define the distracted locations versus the non-distracted locations; non-distracted locations are forward, rearview, right side mirror, left side mirror while the rest of the locations are classified as distracted.

Step Two: 
Identify whether the person is distracted or not distracted at each frame using the criteria explained in the first step.

Step Three:

For each driver, the total number of distracted glances in the last three seconds are summed.

Step Four:

Define the risk due to distraction as a combination of the current glance (weight of 0.2) and the cumulative glance (weight of 0.8 ), as such.;

$$
0.2 *(\text { Current_Glance })+(0.8) *(\text { Cummulative_Glance })
$$

Where current glance is 0 when the driver looks on road and 1 when their eyes are off the road. The cumulative glance is calculated in Step 3.

For considering the driver to be in a state of distraction, any risk value above 2 was considered.

\subsubsection{Cumulative glance current duration and glance eccentricity}

In addition to the criteria observed in the previous two legacy algorithms, this algorithm considers glance eccentricity as a factor in determining the degree of visual demand on the driver. Three ellipses were used to quantify the eccentricity of off-road glances, as done in the 100-Car Study (Dingus et al., 2006). The ellipse was defined in the Table 1. 
Table 1: Definition of the ellipses for each glance location in the CGCDGE algorithm

\begin{tabular}{|c|c|c|}
\hline Ellipse & Location & $\begin{array}{l}\text { Eccentricity } \\
\text { (in glance } \\
\text { radian) }\end{array}$ \\
\hline Ellipse I & $\begin{array}{l}\text { Left forward, right forward, } \\
\text { instrument panel }\end{array}$ & 0.39 \\
\hline Ellipse II & $\begin{array}{l}\text { Center mirror, radio/HVAC, left } \\
\text { mirror }\end{array}$ & 1.12 \\
\hline Ellipse III & All other glances away from road & 2.02 \\
\hline
\end{tabular}

Step One:

For each location, define the degree of eccentricity. The degree of eccentricity is explained in Table 1.

Step Two:

Based on step 1, glances away from the road were penalized. The penalization was done based which ellipse they were categorized in as shown in Table 1. The eccentricity was chosen corresponding to which ellipse the location was categorized in. It is expressed numerically in terms of glance radian as shown in Table 1

Visual Distraction $=\left(6.5758-1 /\left(0.06^{*}\right.\right.$ glance radian +0.152$\left.)\right)$

Values above 32 are indicative of the driver being distracted.

3.1.4 Buffered-cumulative glance current duration and glance eccentricity

This legacy algorithm is also referred to as AttenD. The buffer with a maximum value of two seconds is decremented over time when the driver looks away from the field relevant for 
driving (FRD), which is represented by the intersection between a circle of a visual angle of $90^{\circ}$ and the vehicle windows, excluding the area of the mirrors. When the glance is inside the FRD, the buffer is incremented until the maximum value is reached. There was a latency of $0.1 \mathrm{~s}$ before increasing the buffer after a decrement phase when the driver looks at the mirrors and the speedometer. Additionally, there was a latency of $1 \mathrm{~s}$ before increasing the buffer after a decrement phase when the driver looks anywhere but forward, towards the mirror or the speedometer. The buffer is indicative of the state of the distraction of the driver.

Step One:

Define the locations as found in Table 2.

Table 2: Classification of each glance location for the Buffered-CGCDGE algorithm

\begin{tabular}{|l|l|}
\hline Glance Location & Classification \\
\hline Forward & Field Relevant for Driving (FRD) \\
\hline Rearview Mirror & On Driving (OD) \\
\hline Left Side Mirror & On Driving (OD) \\
\hline Interior Object & Off Road (OR) \\
\hline Right Side Mirror & On Driving (OD) \\
\hline Task displayed on screen & Off Road (OR) \\
\hline Cell Phone & Off Road (OR) \\
\hline Instrument Cluster & On Driving (OD) \\
\hline
\end{tabular}


Step Two:

For each sequence of glances, generate the transition glance, e.g., if the person looks from Forward to the Rearview Mirror, code the transition as "FRD-OD". The sequence is explained in detail in Table 3.

Step Three:

Define the buffer as buffer $=2$. Penalize based on the glance transitions shown in Table 3 .

Table 3: Penalization for glance transitions for the Buffered-CGCDGE algorithm

\begin{tabular}{|l|l|}
\hline Glance Sequence & Buffer Action \\
\hline FRD-FRD & No change in the buffer \\
\hline FRD-OR & 0 \\
\hline FRD-OD & Reduce the buffer by 0.1 with a minimum of \\
\hline OR-OR & Reduce the buffer by 0.1 with a minimum of \\
\hline OR-OD & 0 \\
\hline OR-FRD & Reduce the buffer by 1 with a minimum of 0 \\
\hline OD-OR & Increase the buffer by 0.1 with a delay of $1 ;$ \\
& the maximum value is 2 \\
\hline
\end{tabular}




\begin{tabular}{|l|l|}
\hline OD-OD & Reduce the buffer by 1 with a minimum of 0 \\
\hline OD-FRD & Increase the buffer by 1 with a delay of $1 ;$ the \\
& max value is 2 \\
\hline
\end{tabular}

3.2 Inputs and outputs for each legacy algorithm

Based on the explanation of the four legacy algorithms, the inputs and outputs for each algorithm are defined in Table 4.

Table 4: Input and Output for each legacy algorithm

\begin{tabular}{|l|l|l|}
\multicolumn{1}{|c|}{$\begin{array}{c}\text { Name of the legacy } \\
\text { algorithm }\end{array}$} & \multicolumn{1}{|c|}{ Input } & \multicolumn{1}{c|}{ Output } \\
\hline CG & Glance Location & Total glances \\
\hline CGCD & $\begin{array}{l}\text { Glance Location } \\
\text { Glance Duration }\end{array}$ & $\begin{array}{l}\text { Risk due to } \\
\text { distraction }\end{array}$ \\
\hline CGCDGE & $\begin{array}{l}\text { Glance Location } \\
\text { Glance Duration } \\
\text { Glance Eccentricity }\end{array}$ & Visual Distraction \\
\hline Buffered-CGCDGE & Glance Location & Total Buffer Time for \\
& Glance Duration & the Driver \\
\hline
\end{tabular}




\subsection{Data mining methods}

This study also focuses on advanced methods to ensure better pattern recognition of crashes, which could lead to accurate detection of these events. For example, past research has established distraction detection frameworks based on how drivers perform when subjected to distracting tasks. Research done by Kutila et al.,(2006); Liang et al., (2007); Tango et al., (2013); Wollmer et al., (2011); Yeo et al., (2009) has used SVM as a method for detecting distraction in multiple contexts including camera based classification and even using EEG data.

SVMs are based on statistical learning theory and can be used specifically for classification and pattern recognition. They are also used for understanding the relationships between variables which have linear or non-linear relationships (Furey et al., 2000; Suykens \& Vandewalle, 1999). Apart from linear SVMs, polynomials, splines, radial basis function networks, and multilayer perceptron's SVMs have been successfully applied. In the context of distraction detection, SVM has been applied in the past for classification (Liang et al., 2007; Tango \& Botta, 2013; Wollmer et al., 2011).

For this study, linear SVM was used to classify distracted behavior. The algorithm was trained with glance behavior as well as the vehicle measures for each driver to classify the type of distraction correctly.

\subsection{Data}

\subsubsection{Naturalistic data}


The 100-Car Naturalistic Driving Study (100 Cars) was developed to cement an understanding of driver behaviors and conditions that lead to crashes or near-crash events. This study comprehensively compiled the impact of distracted behavior on crashes/near-crashes as well as environmental factors. It also compiled the impact of eye glance behavior on nearcrash/crash risk.

The data was collected from 2003 to 2004 (Neale et al., 2002) and includes eye glance behavior and vehicular dynamics. The study compiled many extreme cases of driving behavior and performance including: severe fatigue, impairment, judgment error, risk taking, willingness to engage, aggressive driving, and traffic violations. The data collated approximately 2,000,000 vehicle miles with almost 43,000 hours of data wherein 241 primary and secondary drivers participated in the study. Sixty percent of the drivers were male whereas $40 \%$ of the drivers were female. With respect to age, $34 \%$ of the drivers were between the age of $18-24$ years, $35.5 \%$ of the drivers were between the age of $25-44$, and $30.5 \%$ of the drivers were above the age of 45 . Additionally, in the context of driving experience, females reported beginning to drive at an average age of 17.1 years $(\mathrm{SD}=3.51$ years), while males began driving at an average age of 16.2 years $(\mathrm{SD}=1.94$ years). Ethnically, the group of drivers were pre-dominantly Caucasian $(78 \%)$ vs non-Caucasians (22\%). The pool of participants had some sort of education with a majority (41\%) having attended 4 - year college. With respect to traffic violations, almost all the participants reported violations: there were 0.7 violations per participant in the 5 years prior to the beginning of the study in 2003-04.

The baseline database was created specifically for this analysis by randomly selecting a stratified sample of 20,000 6-second segments, referred to as baseline epochs. 


\subsubsection{Driving simulator data}

Past research has found that the novice drivers have increased crash risk compared to experienced drivers when subjected to distractions like dialing a cell phone, eating, or drinking (Klauer et al., 2014). In comparison to past studies on teen driving behavior, the focus was on recreating a driving scenario wherein the young drivers were exposed to typical distracted scenarios. To do that, driving with passengers was considered a typical scenario as it has been identified that the influence of passengers does impact driver performance (Ouimet et al., 2013).

\subsubsection{Definition of an event}

For the events that occurred during the simulator study, the definitions used were the same as defined for the 100 cars study:

- Any physical contact between the subject vehicle and another vehicle, fixed object, pedestrian, cyclist, animal, etc., as assessed by either the lateral or longitudinal accelerometers was termed a crash. The most predominant type of crash in the simulator data was a rear end crash.

- A Near-crash was a conflict situation requiring a rapid, severe, evasive maneuver to avoid a crash.

\subsection{Experimental Design}

\subsubsection{Recruitment}


There were 24 pairs of participants that were recruited through communications (online and print advertisements) on campus at University of Massachusetts, Amherst. The age group selected was between 18-20 years. All participants needed to have a valid driving license of the United States. Each participant was recruited who satisfied the criteria and were asked to participate with a friend/colleague/partner.

\subsubsection{Apparatus}

For this study, the driving simulator at the Human Performance Laboratory, University of Massachusetts, Amherst was used. It is a fixed base driving simulator which uses Realtime Technologies, Inc. (RTI) simulation software (Figure 5). The simulator is a full car cab (4-door 2013 Ford Fusion) with nine visual channels. The five forward channels plus the rear channel create a 330-degree field-of-view (FOV). The side-view mirrors, virtual dash, and 17-inch touch screen center stack are simulated with LCD panels. A 5.1 channel audio system external to the car cab provides the environmental sounds such as traffic, passing vehicles, and road noise. An internal audio system provides the engine sounds and vibrations, as well as pre-programmed voice commands and any other scripted sounds. The participant in the automobile can move through the virtual world using the vehicle controls as if in a real automobile and the visual representation of the virtual roadway changes appropriately in response to drivers' actions. Vehicle data is continuously collected at a frequency of $96 \mathrm{~Hz}$. 


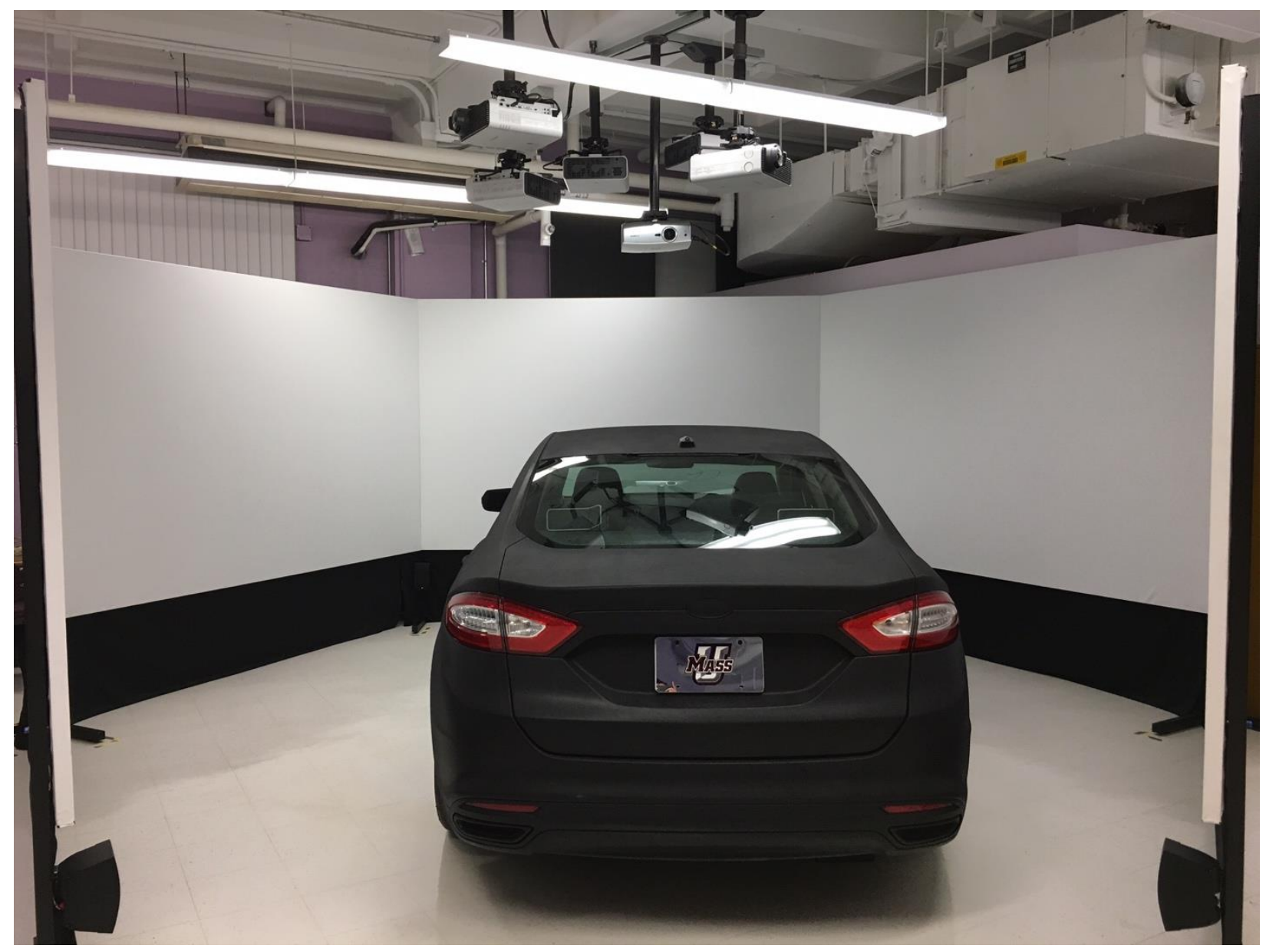

Figure 5: The driving simulator

The eye tracker, a Mobile Eye XG by Applied Science, allows one to track and record eye movements to study behavioral scanning patterns. The eye tracker is comprised of a pair of safety goggles equipped with two lightweight cameras: one to track eye movements and the other to capture the scene that the user sees. The device is worn by each participant while they are driving as shown in Figure 6. The two videos are recorded, processed, and interleaved on a device that outputs a video file with cross-hairs displaying the drivers gaze and the associated coordinate data in a separate data file. The eye tracking data was recorded at a frequency of 33 HZ. 


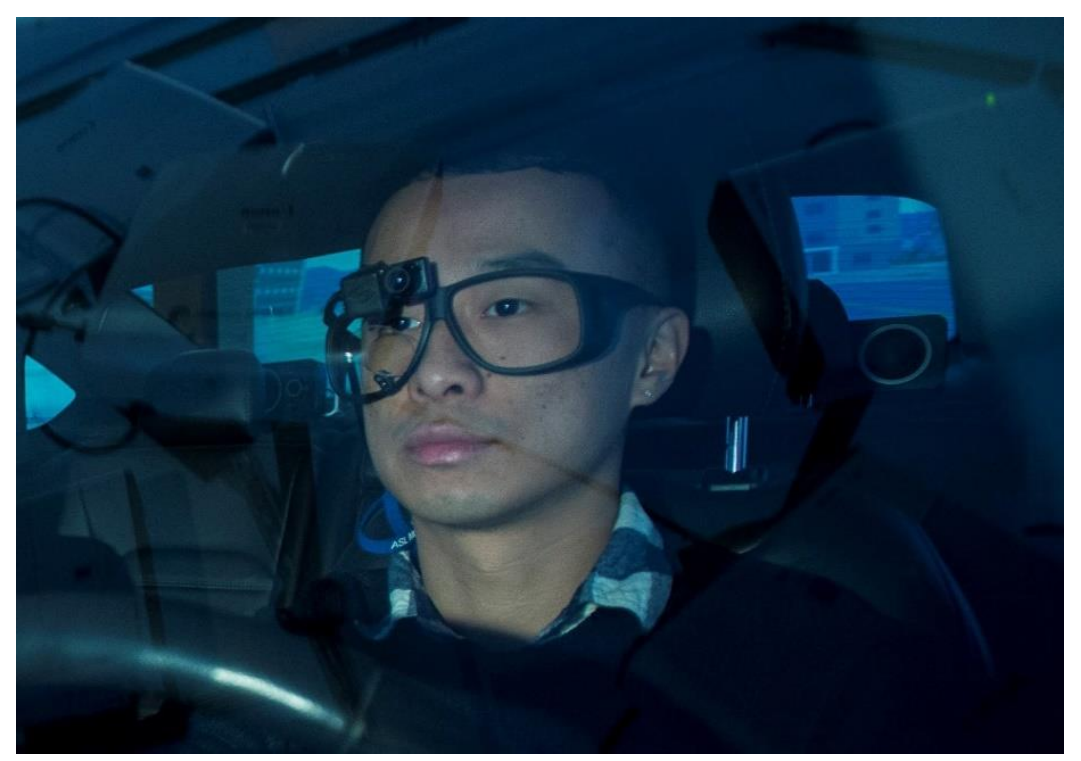

Figure 6: The eye tracker used to record glance behavior

\subsection{Procedure}

Each pair of participants was asked to participate in the drive for 90 minutes. Before the experiment, participants gave consent. The consent forms were approved by the Institutional Review Board (IRB). Next, they were asked whether they had any pre-conditions that would inhibit them from driving. Afterwards, they were given instructions about the experiment. Participants were given a test-drive to acclimate them to the driving simulator.

The driving environment was a highway scenario like I-93 in Western Massachusetts. The road included 4 straight stretches and 4 curves with medium to low traffic density. There were two points on each road where there was more than the regular traffic density. After the participants completed their drives, they were asked to fill out the post drive questionnaire which included the sensation seeking questionnaire, driver behavior questionnaire, their driving history, and demographic information. Each participant was compensated \$25. 


\subsection{Distracting tasks}

There were three distraction tasks: visual, cognitive and combined distractions. The control condition was no distraction. The visual task was dialing a phone number displayed on the screen (Figure 7). While participants drove alone, the cognitive task was the Delta Flight Information task (Angell et al., 2006), where the driver is asked questions after listening to Delta Flight information. When the participants drove with a passenger, they were asked to perform a chain task, which was a variant of the task conducted by Crundall et al. (2005). The participants were asked to say a word and the other person would add another word to previous word, thereby creating a chain of words. In the opposing drive (where the driver and passenger switch roles), a similar task was completing using double digit numbers. The combined visual and cognitive task required a display of arrows in random directions (Figure 8). The participants were asked to answer two questions based on the arrow image, similar to Liang \& Lee (2010).

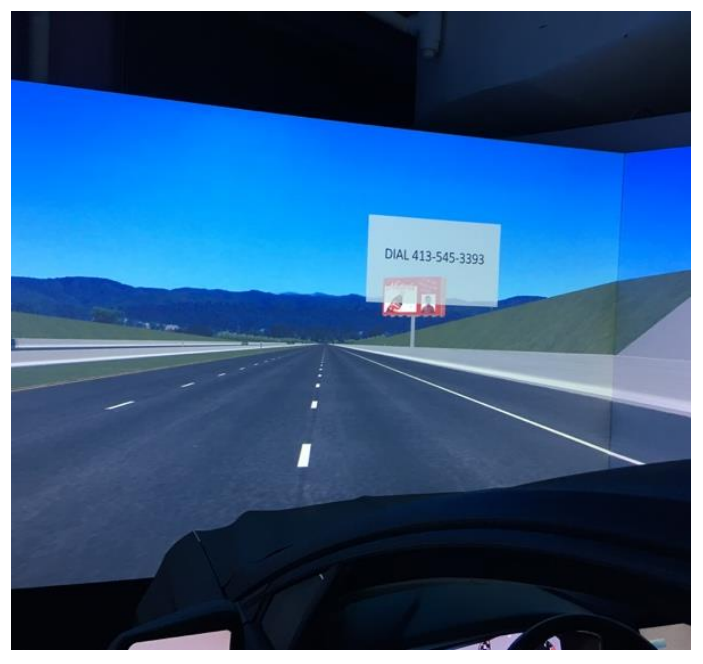

Figure 7: Visual distraction task

To overcome any learning effects, all tasks were counterbalanced. Additionally, all phone numbers were changed in each drive, a separate set of questions were asked for the Delta Flight 
Information, and a unique randomized set of arrows were used so the participant could not anticipate the answers before each drive.

\begin{tabular}{|c|c|c|c|c|}
\hline & 1 & 2 & 3 & 4 \\
\hline$A$ & $\downarrow$ & $\lambda$ & 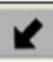 & $\uparrow$ \\
\hline$B$ & $\lambda$ & $\Rightarrow$ & $y$ & $y$ \\
\hline C & $\uparrow$ & $\boldsymbol{K}$ & $\leftarrow$ & $\downarrow$ \\
\hline D & $k$ & $\leftarrow$ & $\Rightarrow$ & $\boldsymbol{K}$ \\
\hline
\end{tabular}

Figure 8: Combined distraction task

\subsection{Eye tracking}

With each eye tracking video, two independent coders examined each frame to identify where the driver glanced at each moment of time. The areas of interests are defined in Table 5.

Table 5: Definition of each area of interest

\begin{tabular}{|l|l|}
\hline Area of Interest & Description \\
\hline Forward & Looking in front with eyes on the road or \\
on a car
\end{tabular}




\begin{tabular}{|l|l|}
\hline Interior Object & $\begin{array}{l}\text { Looking anywhere except the } \\
\text { aforementioned areas (e.g., center stack, } \\
\text { passenger etc.) }\end{array}$ \\
\hline
\end{tabular}

The glances were coded for these areas of interest using BORIS (Friard \& Gamba, 2016). In case of a conflict, additional researchers examined the coding discrepancy.

3.9 Vehicle data

The vehicle data was captured by the high-fidelity simulator. The data was pre-processed, and six dependent variables were extracted, including: time, acceleration $(\mathrm{m} / \mathrm{s} 2)$, lateral acceleration $(\mathrm{m} / \mathrm{s} 2)$, offset in lane position (meters), degree of rotation of the steering wheel (degrees), velocity of the vehicle (mph), lateral velocity (mph), and vertical velocity of the vehicle (mph). The data was compiled for all participants using $R$ statistical analysis software ( $R$ Core Team, 2017). 


\section{CHAPTER 4}

\section{RESULTS}

This section looks at the comparison of the results obtained from the legacy algorithms on both data sources. While interpretation and validation of these legacy algorithms is the primary objective, exploring distracted driving behavior and the glance behavior leading up to the crash like event is also important and of interest. In this section, glance behavior for both data sets is explored and then used to evaluate the legacy algorithms. Along with the evaluation, machine learning methods that detect distraction are also explored.

\subsection{Exploratory analysis of glance behavior}

For the 100-cars study, only participants who were involved in crash like events due to distraction have been considered. After selecting those relevant to this study, the start and end time of the glance location for each drive was reduced to a frequency of $1 \mathrm{~Hz}$. Upon data preprocessing, the glance behavior of each drive was analyzed 5 seconds before the precipitating event. This can be attributed to the fact the past research has indicated that 6 to 8 seconds of reaction time is required to avoid a crash (Lamble, Laakso, \& Summala, 1999). In the 100 cars study, it is observed that $74.30 \%$ of glances were towards the forward roadway. Another $16.46 \%$ of glances were distributed between drivers looking at interior objects (including conversing with passengers), cell phones, and having their eyes closed. The remaining $9.24 \%$ of the glances are directed towards looking left, right and rearview mirrors, which could be attributed to drivers attempting to maintain situational awareness leading up to, during, and after a crash like event. This distribution is shown in Figure 9. 


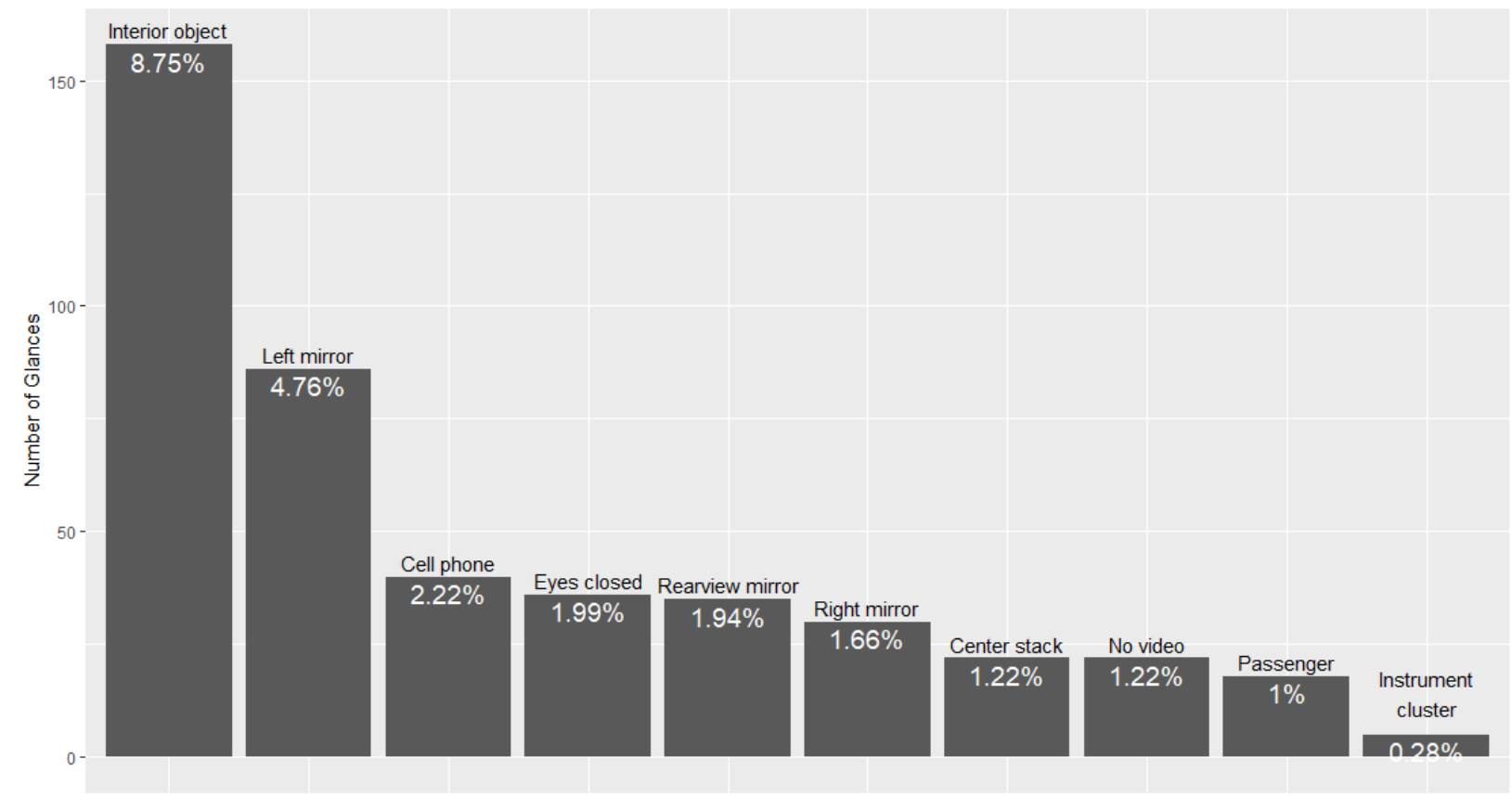

Figure 9: Glance locations (excluding Forward) for the distracted participants 5 seconds before the crash in the 100 Cars dataset 


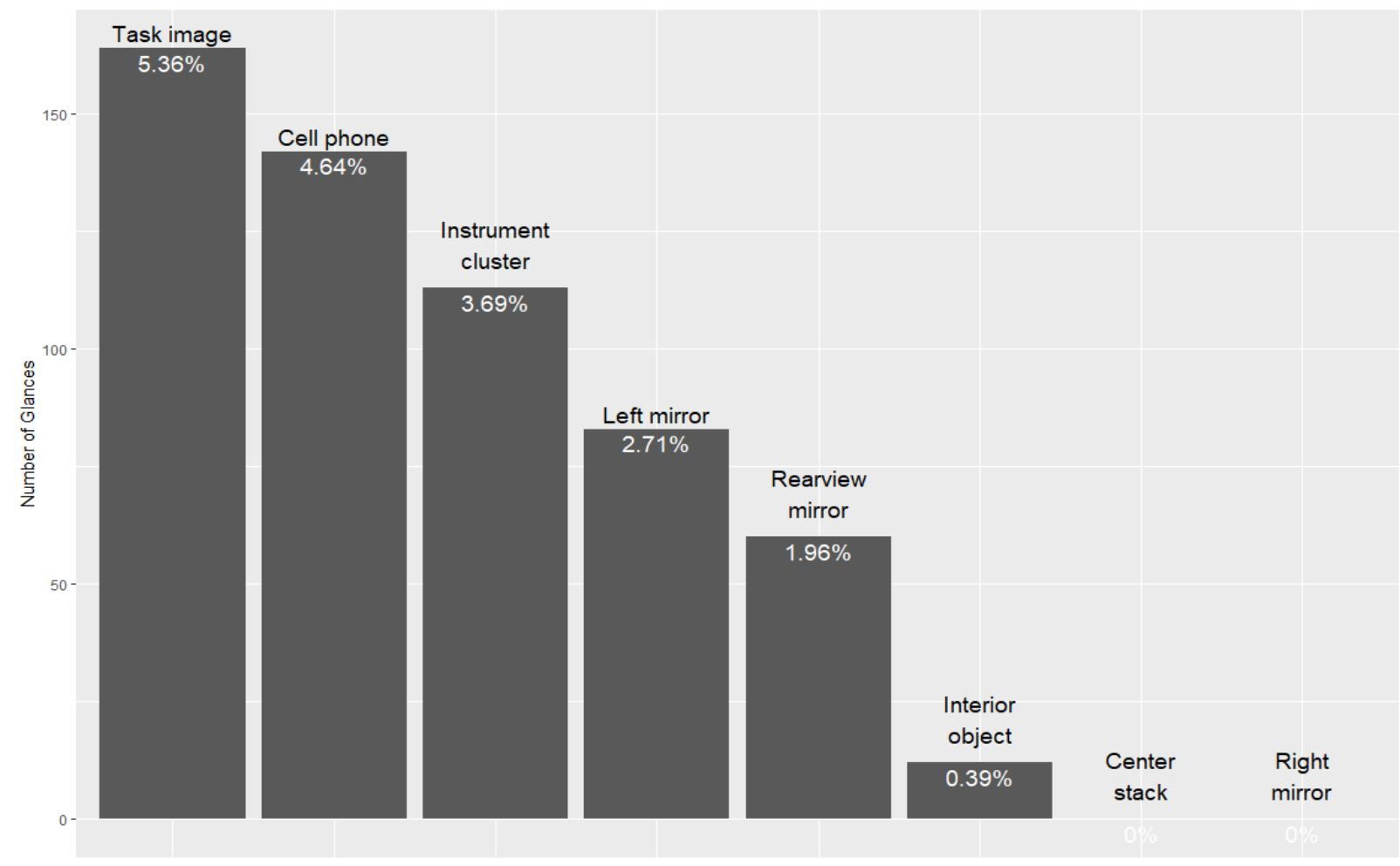

Figure 10: Glance locations (excluding Forward) for the distracted participants 5 seconds before the crash for the simulator data

Similar findings were observed from the simulator data: the participant is observed to be looking forward more than $90 \%$ of the time. For the remaining $10 \%$, the driver is completing the visually distracting tasks, which is shown in Figure 10. While the results may seem counterintuitive that during crash events the person looks forward, it is interesting to observe that their glances off the road might influence the crash like scenario. This provides preliminary evidence that crash likelihood could increase due to glances away from the road. Additional analysis around sequence of glances leading up to the crash could provide more evidence to the existing analysis and could help further understand eye glance behavior. 


\subsection{Evaluation of the legacy algorithms}

Next, the four legacy algorithms, the Current Glance (CG), the Current Glance Cumulative Duration (CGCD), the Current Glance Cumulative Duration and Glance Eccentricity (CGCDGE), and Buffered-CGCDGE, were evaluated. The legacy algorithms were implemented using the criteria explained in the Methodology section. The metric used for the purposes of explaining the predicting power of the algorithm is Odds Ratio (OR). An odds ratio (OR) is a measure of association between an exposure and an outcome. The OR represents the odds that an outcome will occur given a condition, compared to the odds of the outcome occurring in the absence of that condition. If $\mathrm{OR}>1$, then the condition has a higher effect on the outcome; if $\mathrm{OR}<1$, then the condition has a lower effect on the outcome; and if $\mathrm{OR}=1$, then the condition has no effect on the outcome. An example would be the odds of a driver being involved in a crash like event where the condition is that the driver is distracted, compared to the driver being involved in a crash if they are not distracted. In this example, an OR $>1$ indicates that the drivers is more likely to be in a crash if they are distracted.

All legacy algorithms can detect distraction where the OR of predicting distraction is greater than 1 for both datasets (Figure 11). It may be noted that CGCDGE is not included in Figure 12 because the odds ratios were $\sim 20,000$, which is exaggerated due to the high penalization rate for task completion. The highest odds are for legacy algorithms that account for the glance history, which is in-line with expectations. 


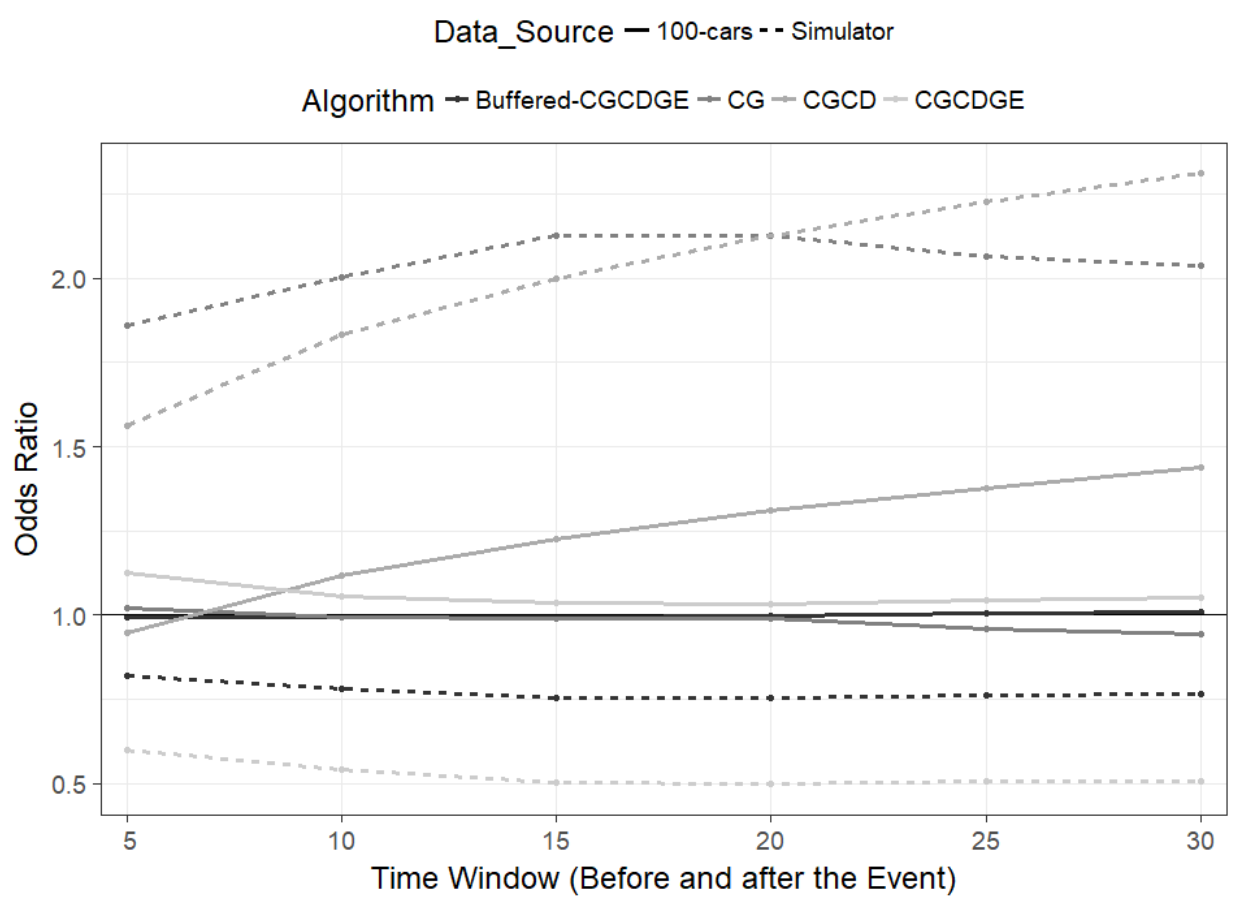

Figure 11: Odds ratios for detecting distraction for different time windows

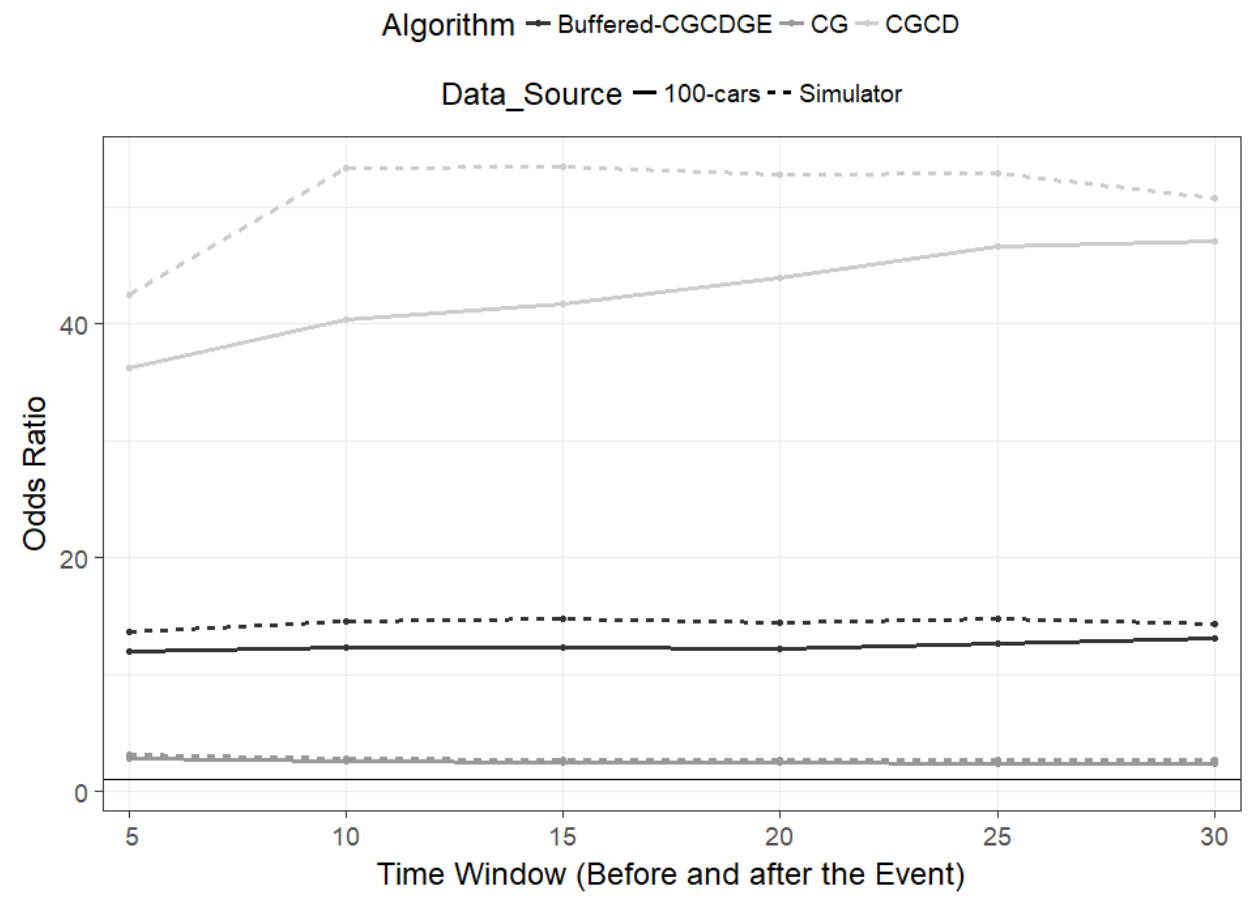

Figure 12: Odds ratios for detecting an event for different time windows 
As far as event detection is concerned, different results were observed. CG outperforms all the other legacy algorithms in terms of event detection as shown in Figure 12. This could be attributed to the design of CG which considers distraction as "looking away from the road for 2 seconds in a 6 second window" (Klauer et al., 2010) where the event occurs in the $5^{\text {th }}$ second. Additionally, it is interesting to note that the odds improve with an increasing time window. This could be attributed to having more data for detection and thus providing better odds in detecting a event. Also, an unexpected result was observed for the Buffered-CGCDGE on the simulator data where it outperforms CG until the 15 second window. While it is difficult to pinpoint one cause for this, Buffered-CGCDGE does include the most parameters out of all the legacy algorithms, which could lead to the odds being exacerbated due to previous glance behavior.

\subsection{Impact of distraction on vehicle measures}

For this study, the vehicle measures obtained from the simulator data was used to understand driver behavior when subjected to different distractions. As discussed in the methodology section, the participants were subjected to three different types of distractions - visual, cognitive and combined (visual and cognitive). The measures used for this analysis are velocity, acceleration (or deceleration), lane offset, lateral velocity, and steering wheel angle.

\subsection{Detection of distraction using support vector machines}

To select the variables for training and validating the machine learning algorithm, Pearson's correlation was used. In Table 6 below, the cross-correlation of all the variables is displayed. The values highlighted in green indicate a strong positive or negative correlation between the variables in the respective row and column. Apart from lane offset, all other 
variables have a strong correlation between each other. Based on this information, the variables chosen for model development were: velocity (measured in $\mathrm{mph}$ ), lateral velocity (measured in mph), and steering wheel movements (measuring the rotation of the steering wheel in radians).

Table 6: Correlation matrix for vehicle measures

\begin{tabular}{|l|r|l|l|r|r|}
\hline & \multicolumn{1}{l|}{$\begin{array}{l}\text { Lateral } \\
\text { Acceleration }\end{array}$} & \multicolumn{1}{l|}{$\begin{array}{l}\text { Steering } \\
\text { Wheel }\end{array}$} & \multicolumn{1}{l}{$\begin{array}{l}\text { Lateral } \\
\text { Velocity }\end{array}$} & Velocity & Lane Offset \\
\hline $\begin{array}{l}\text { Lateral } \\
\text { Acceleration }\end{array}$ & 1.0000000 & -0.4633717 & -0.7432685 & 0.1009258 & -0.1123495 \\
\hline $\begin{array}{l}\text { Steering } \\
\text { Wheel }\end{array}$ & -0.4633717 & 1.0000000 & 0.5453632 & 0.4193530 & 0.1036230 \\
\hline $\begin{array}{l}\text { Lateral } \\
\text { Velocity }\end{array}$ & -0.7432685 & 0.5453632 & 1.0000000 & 0.2616517 & 0.1046672 \\
\hline Velocity & 0.1009258 & 0.4193530 & 0.2616517 & 1.0000000 & -0.0107414 \\
\hline & -0.1123495 & 0.1036230 & 0.1046672 & -0.0107414 & 1.0000000 \\
\hline
\end{tabular}

To arrive at the best possible classifier, only data 60 seconds before and after the event was used. Upon filtering the data for this criterion, the four variables were standardized by using the scale function (R Core Team, 2017) which converts all the variables to have a mean of 0 and a standard deviation of 1 . Next, the classifier was trained with a sample of 1000 observations. The validation was carried out with another unique 1000 observations. The analysis was done in the R programming language (R Core Team, 2017) using the package "e1071” (Meyer, Dimitriadou, Hornik, Weingessel, \& Leisch, 2017). The package was chosen due to its simplicity and abundant online resources.

In both training and validation, it appears that the models cannot detect the state of distraction. Table 7 below shows the best accuracy, precision, and recall values from all 1000 iterations. 
Table 7: Distraction detection results for machine learning methods

\begin{tabular}{|l|r|r|l|r|r|r|}
\cline { 2 - 8 } \multicolumn{1}{c|}{} & \multicolumn{3}{c|}{ Training } & \multicolumn{3}{c|}{ Validating } \\
\hline Distraction & Accuracy & Precision & Recall & Accuracy & Precision & Recall \\
\hline Visual Distraction & 0.603 & 0.5734 & 0.53478 & 0.503 & 0.4568 & 0.4261 \\
\hline $\begin{array}{l}\text { Cognitive } \\
\text { Distraction }\end{array}$ & 0.534 & 0.3428 & 0.3408 & 0.564 & 0.3456 & 0.3731 \\
\hline $\begin{array}{l}\text { Combined } \\
\text { Distraction }\end{array}$ & 0.579 & 0.322 & 0.3717 & 0.557 & 0.3101 & 0.3429 \\
\hline
\end{tabular}

The results show that while using pattern recognition or data mining methods, vehicle measures may not be enough in detecting distraction. Additionally, when glance locations were included in the model development, the results were poor.

All in all, this research provides validation of legacy algorithms and attempts to propose a better solution for the detection of distraction. The results indicate that distraction can be detected best with glance history and glance eccentricity. However, event detection is best when only considering glance history. Additionally, this research indicated that data mining approaches do not outperform legacy approaches in terms of detecting of distraction. While there are no definitive reasons, it is possible that the current state alone may not be enough to consider the state of distraction. In the future, cumulative glances in combination with driving behavior should be considered to detect the likelihood of distraction or event. 


\section{CHAPTER 5}

\section{DISCUSSION}

This research has addressed the comparison of four legacy algorithms over two different data sources. While research in the past has evaluated these legacy algorithms (Liang et al., 2012; Seppelt et al., 2017), the novelty of the research lies in comparison across two different data sources. The results indicate that these legacy algorithms provide an efficient way to detect distraction, which is expected based on their focus on classifying glances away from the road as distraction. However, an even more interesting finding is the way these legacy algorithms can detect events, which have implications on predicting whether the driver will crash (Donmez et al., 2008). As explained in the background section, these results connect to the multiple resource theory (MRT). Additionally, the relatively inefficient distraction detection using SVMs using vehicle data explains the limitations of relying on only vehicle data to detect driver state. This section looks to address these findings, their implications, and their limitations within the scope of this study.

5.1 Detection of distraction and events due to the legacy algorithms

Based on the results, it is evident that the performance of the four legacy algorithms is excellent with respect to detecting distraction. This has been consistent with past research in terms of the odds ratios being similar as found by Liang et al., 2012. Additionally, it was found that the Cumulative Glance algorithm efficiently detects the likelihood of an event, which it is supposed to do by design. This implies that legacy algorithms that detect the likelihood of an event might indirectly detect the likelihood of a distraction. However, legacy algorithms that 
detect the likelihood of distraction may not detect events. This can explain the relatively poor performance of legacy algorithms other than CG in detecting the likelihood of an event.

The distraction impairs the drivers' situational awareness because the attention allocation is towards a non-driving objects, which leads the driver to be negligent (Regan et al., 2011). Thus, a key finding from these results is the consideration of the previous glances when determining the state of distraction. Additionally, the high odds of CGCDGE confirms the extent to which the eyes are off the road can detect the state of distraction.

Another aspect to consider is the comparable results for these legacy algorithms on the naturalistic as well as simulator data. This has a huge implication as far as distraction detection is concerned. More specifically, while past research has focused on either the naturalistic or simulator data, no study has explored both sources of data together. The results of this validation study indicate the applicability of these legacy algorithms in distraction detection across different data sources.

\subsection{Vehicle data in detecting distraction}

While the eye glance data and the legacy algorithms were efficient in detecting the level of distraction, the vehicle data was not as useful. The SVM, which relies on vehicle data, does not perform well in detecting the state of distraction. There are several possible reasons for this lackluster result. First, this method may not be useful when considering vehicle data. For the simulator data, no major differences between the distracted state and the non-distracted state in terms of the vehicle measures were observed. Additionally, the major difference between SVM and the legacy algorithms is cumulative driving measures or glances. The addition of cumulative glances as input to the SVM may improve its' results. 
Second, while SVM is a good approach, it is not the only machine learning approach that is applicable for detecting distraction. Exploring alternate methods like decision trees or unsupervised learning methods could possibly yield better results in terms of classifying the state of the driver. Another advantage of using these methods could be the possible increase the accuracy of distraction detection with an increase in data. For example, Kircher et al. (2010) used measures like steering entropy, variance in speed, and throttle behavior in predicting visual distraction. Tango (2013) used visual behavior as well as lane performance metrics as a factor in detecting distraction using SVMs. Additionally, studies done by Caird et al., (2018) used metrics like weather, road type, and traffic as a factor in predicting an event. Future studies could combine legacy algorithms along with different machine learning approaches to provide robust methods for detection and prediction.

\subsection{Limitations and future work}

The definition of distraction as stated in this research deems the driver unable to focus on activities critical for ensuring safe driving when subjected to distracting tasks. While this definition does establish a fundamental understanding of driving behavior, it has limitations. For example, if a driver is distracted, eye glance measures may not be sufficient in indicating the state of distraction. The driver could potentially not be engaged in the distracting task if their attention divided. More research is needed to clearly delineate periods of distraction versus no distraction. Future work should consider multiple measures of a "ground truth" of distraction.

While validating legacy algorithms on naturalistic data is an efficient way to understand performance, the data used was recorded in 2003-04, which is approximately 15 years ago. Validating these legacy algorithms on newer data sources would be a better way to understand 
efficiency. Data sources like SHRP2 (Campbell, 2012) are perfect sources for validation, however they are not publicly available. Similarly, simulator data from a different study might produce other interesting findings. These approaches could help validate this study and could potentially put forth a better method of detecting driver distraction.

In this study, the vehicle measures have only been used for classifying the type of distraction. While this establishes a useful baseline in understanding driving behavior, the use of vehicle measures for event classification is a logical next step.

Although this study looks at distraction from a holistic point of view, one limitation is the application of this information. Legacy algorithms can be used to design feedback systems to mitigate distraction (Donmez et al., 2008). This feedback system would prevent the driver from being distracted and provide them with warnings to adapt their behavior in near-real time. For these circumstances, it is important to understand the efficiency of these legacy algorithms (i.e., how long it takes to detect distraction) in providing real time feedback. This would be building block for designing feedback systems in the future. 


\section{BIBLIOGRAPHY}

Angell, L., Auflick, J., Austria, P. a., Kochhar, D., Tijerina, L., Biever, W., ... Kiger, S. (2006). Driver workload metrics project: Task 2 final report. Security, (November), 460.

Caird, J. K., Simmons, S. M., Wiley, K., Johnston, K. A., \& Horrey, W. J. (2018). Does Talking on a Cell Phone, With a Passenger, or Dialing Affect Driving Performance? An Updated Systematic Review and Meta-Analysis of Experimental Studies. Human Factors: The Journal of the Human Factors and Ergonomics Society, 60(1), 101-133. https://doi.org/10.1177/0018720817748145

Campbell, K. L. (2012). The SHRP 2 Naturalistic Driving Study. Transportation Research News, 30-35. Retrieved from http://onlinepubs.trb.org/onlinepubs/trnews/trnews282SHRP2nds.pdf

Crundall, D., Bains, M., Chapman, P., \& Underwood, G. (2005). Regulating conversation during driving: A problem for mobile telephones? Transportation Research Part F: Traffic Psychology and Behaviour, 8(3), 197-211. https://doi.org/10.1016/j.trf.2005.01.003

Dingus, T. a., Klauer, S. G., Neale, V. L., Petersen, A., Lee, S. E., Sudweeks, J., ... Knipling, R. R. (2006). The 100-Car naturalistic driving study phase II - Results of the 100-Car field experiment. Dot Hs 810 593, (April), No. HS-810 593. https://doi.org/DOT HS 810593

Donmez, B., Boyle, L. N., \& Lee, J. D. (2007). Safety implications of providing real-time feedback to distracted drivers. Accident Analysis and Prevention, 39(3), 581-590. https://doi.org/10.1016/j.aap.2006.10.003

Donmez, B., Boyle, L. N., \& Lee, J. D. (2008). Mitigating driver distraction with retrospective and concurrent feedback. Accident Analysis and Prevention, 40(2), 776-786.

https://doi.org/10.1016/j.aap.2007.09.023

Donmez, B., Boyle, L. N., Lee, J. D., \& Mcgehee, D. V. (2006). Drivers ' attitudes toward imperfect distraction mitigation strategies, 9, 387-398. https://doi.org/10.1016/j.trf.2006.02.001

Engström, J., \& Mårdh, S. (2007). SafeTE Final Report, Publication No. 2007: 36. Vägverket, Sweden.

Engström, J., Markkula, G., \& Trent, V. (2009). Attention selection and task interference indriving: an action-oriented view. In 1st International Conference on Driver Distraction and Inattention (DDI 2009) Chalmers University of Technology, SwedenSAFER Vehicle and Traffic Safety CentreINRETS-ARCUEIL, FRANCE.

Engström, J., Victor, T., \& Markkula, G. (2013). Attention selection and multitasking in everyday driving: A conceptual model.

Fell, J. C., \& Voas, R. B. (2006). Mothers Against Drunk Driving (MADD): The first 25 years. Traffic Injury Prevention, 7(3), 195-212. https://doi.org/10.1080/15389580600727705

Fletcher, L., \& Zelinsky, A. (2007). Driver state monitoring to mitigate distraction, 487-523. 
Friard, O., \& Gamba, M. (2016). BORIS: a free, versatile open-source event-logging software for video/audio coding and live observations. Methods in Ecology and Evolution, 7(11), 1325-1330. https://doi.org/10.1111/2041-210X.12584

Furey, T. S., Cristianini, N., Duffy, N., Bednarski, D. W., Schummer, M., \& Haussler, D. (2000). Support vector machine classification and validation of cancer tissue samples using microarray expression data. Bioinformatics, 16(10), 906-914.

Goodwin, A. H., O’Brien, N. P., \& Foss, R. D. (2012). Effect of North Carolina's restriction on teenage driver cell phone use two years after implementation. Accident Analysis and Prevention, 48, 363-367. https://doi.org/10.1016/j.aap.2012.02.006

Hancock, P. A., Mouloua, M., \& Senders, J. W. (2009). On the philosophical foundations of the distracted driver and driving distraction.

Horrey, W. J., \& Wickens, C. D. (2004). Driving and Side Task Performance: The Effects of Display Clutter, Separation, and Modality. Human Factors: The Journal of the Human Factors and Ergonomics Society, 46(4), 611-624. https://doi.org/10.1518/hfes.46.4.611.56805

Ibrahim, J. K., Anderson, E. D., Burris, S. C., \& Wagenaar, A. C. (2011). State laws restricting driver use of mobile communications devices: Distracted-driving provisions, 19922010. American Journal of Preventive Medicine, 40(6), 659-665. https://doi.org/10.1016/j.amepre.2011.02.024

Kahn, C. A., \& Gotschall, C. S. (2015). The economic and societal impact of motor vehicle crashes, 2010 (Revised). Annals of Emergency Medicine, 66(2), 194-196. https://doi.org/10.1016/j.annemergmed.2015.06.011

Kim, H., Kwon, S., Heo, J., Lee, H., \& Chung, M. K. (2014). The effect of touch-key size on the usability of In-Vehicle Information Systems and driving safety during simulated driving. Applied Ergonomics, 45(3), 379-388. https://doi.org/10.1016/j.apergo.2013.05.006

Kircher, K., \& Ahlstrom, C. (2010). Predicting visual distraction using driving performance data. Annals of Advances in Automotive Medicine, 54th Annual Scientific Conference of the Association for the Advancement of Automotive Medicine, 54, 333-42. Retrieved from http://www.pubmedcentral.nih.gov/articlerender.fcgi?artid=3242543\&tool=pmcentrez\&ren dertype $=$ abstract\%5Cnhttp://www.ncbi.nlm.nih.gov/pubmed/21050615\%5Cnhttp://www.pu bmedcentral.nih.gov/articlerender.fcgi?artid=PMC3242543

Kircher, K., \& Ahlström, C. (2009). Issues related to the driver distraction detection algorithm AttenD. 1st International Conferences on Driver Distraction and Inattention, 1-15.

Kircher, K., \& Ahlström, C. (2013). The driver distraction detection algorithm AttenD.

Kircher, K., Kircher, A., \& Ahlström, C. (2009). Results of a field study on a driver distraction warning system. 
Klauer, S. G., Dingus, T. A., Neale, V. L., Sudweeks, J., \& Ramsey, D. (2009). Comparing RealWorld Behaviors of Drivers With High versus Low Rates of Crashes and Near-Crashes, (February), 1-204.

Klauer, S. G., Guo, F., Simons-Morton, B. G., Ouimet, M. C., Lee, S. E., \& Dingus, T. A. (2014). Distracted driving and risk of road crashes among novice and experienced drivers. New England Journal of Medicine, 370(1), 54-59.

Klauer, S. G., Guo, F., Sudweeks, J. D., \& Dingus, T. a. (2010). An analysis of driver inattention using a case-crossover approach on 100-car data: Final report. U.S. Department of Transportation: National Highway Traffic Safety Administration, (May), 148.

Kutila, M., Jokela, M., Markkula, G., \& Rué, M. R. (2006). Driver distraction detection with a camera vision system. Proceedings - International Conference on Image Processing, ICIP, 6(3 1), 201-204. https://doi.org/10.1109/ICIP.2007.4379556

Lamble, D., Laakso, M., \& Summala, H. (1999). Detection thresholds in car following situations and peripheral vision: Implications for positioning of visually demanding in-car displays. Ergonomics, 42(6), 807-815. https://doi.org/10.1080/001401399185306

Lee, J. D. (2008). Fifty Years of Driving Safety Research. (Cover story). Human Factors, 50(3), 521-528. https://doi.org/10.1518/001872008X288376.

Lee, J., Moeckli, J., Brown, T. L., Roberts, S., Victor, T., Marshall, D., ... Nadler, E. (2013). Detection of driver distraction using vision-based algorithms. Proc. 23rd Enhanced Safety Veh. Conf.

Liang, Y. (2009). Detecting driver distraction. ProQuest Dissertations and Theses, 3356220, 152. Retrieved from http://ezproxy.net.ucf.edu/login?url=http://search.proquest.com/docview/304900929?accou ntid=10003\%5Cnhttp://sfx.fcla.edu/ucf?url_ver=Z39.88-

2004\&rft_val_fmt=info:ofi/fmt:kev:mtx:dissertation\&genre=dissertations $+\&+$ theses $\&$ sid $=\mathrm{P}$ roQ:ProQuest+Dissertations+\&+T

Liang, Y., \& Lee, J. D. (2010). Combining cognitive and visual distraction: Less than the sum of its parts. Accident Analysis and Prevention, 42(3), 881-890.

https://doi.org/10.1016/j.aap.2009.05.001

Liang, Y., Lee, J. D., \& Yekhshatyan, L. (2012). How Dangerous Is Looking Away From the Road? Algorithms Predict Crash Risk From Glance Patterns in Naturalistic Driving. Human Factors: The Journal of the Human Factors and Ergonomics Society, 54(6), 1104-1116. https://doi.org/10.1177/0018720812446965

Liang, Y., Reyes, M. L., \& Lee, J. D. (2007). Real-time detection of driver cognitive distraction using support vector machines. IEEE Transactions on Intelligent Transportation Systems, 8(2), 340-350. https://doi.org/10.1109/TITS.2007.895298

MacLean, A. W., Davies, D. R. T., \& Thiele, K. (2003). The hazards and prevention of driving while sleepy. Sleep Medicine Reviews, 7(6), 507-521. https://doi.org/10.1016/S10870792(03)90004-9 
McDonald, a. D., Lee, J. D., Schwarz, C., \& Brown, T. L. (2013). Steering in a Random Forest: Ensemble Learning for Detecting Drowsiness-Related Lane Departures. Human Factors: The Journal of the Human Factors and Ergonomics Society, 56(5), 986-998. https://doi.org/10.1177/0018720813515272

Meyer, D., Dimitriadou, E., Hornik, K., Weingessel, A., \& Leisch, F. (2017). e1071: Misc Functions of the Department of Statistics, Probability Theory Group (Formerly: E1071), TU Wien. Retrieved from https://cran.r-project.org/package=e1071

Michon, J. (1985). A critical view of driver behavior models: what do we know, what should we do? Human Behavior and Traffic Safety, 485-520. https://doi.org/10.1007/978-1-46132173-6

National Safety Council. (2013). Crashes Involving Cell Phones: Challenges of Collecting and Reporting Reliable Crash Data, 1-12. Retrieved from http://www.nsc.org/DistractedDrivingDocuments/NSC-Under-Reporting-White-Paper.pdf

Neale, V. L., Klauer, S. G., Knipling, R. R., Dingus, T. a., Holbrook, G. T., \& Petersen, A. (2002). The 100 Car Naturalistic Driving Study Phase I - Experimental Design. US DOT, National Highway Traffic Safety Administration, (December). https://doi.org/DOT HS 808 536

NHTSA. (2017). TRAFFIC SAFETY FACTS 2016 Fatal Motor Vehicle Crashes: Overview, (October), 1-9. https://doi.org/DOT HS 812456

Ouimet, M. C., Pradhan, A. K., Simons-Morton, B. G., Divekar, G., Mehranian, H., \& Fisher, D. L. (2013). The effect of male teenage passengers on male teenage drivers: Findings from a driving simulator study. Accident Analysis and Prevention, 58, 132-139. https://doi.org/10.1016/j.aap.2013.04.024

Pohl, J., Birk, W., \& Westervall, L. (2007). A driver-distraction-based lane-keeping assistance system, 221, 541-552. https://doi.org/10.1243/09596518JSCE218

R Core Team. (2017). R: A Language and Environment for Statistical Computing. Vienna, Austria. Retrieved from https://www.r-project.org/

Regan, M. A., Hallett, C., \& Gordon, C. P. (2011). Driver distraction and driver inattention: Definition, relationship and taxonomy. Accident Analysis and Prevention, 43(5), 17711781. https://doi.org/10.1016/j.aap.2011.04.008

Schwarz, C., Brown, T., Lee, J., Gaspar, J., \& Kang, J. (2016). The detection of visual distraction using vehicle and driver-based sensors. SAE Technical Paper, (April), 2016-01-0114. https://doi.org/10.4271/2016-01-0114.Copyright

Senders, J. W., Kristofferson, A. B., Levison, W. H., Dietrich, C. W., \& Ward, J. L. (1967). The attentional demand of automobile driving. Highway Research Record, (195). 
Seppelt, B. D., Seaman, S., Lee, J., Angell, L. S., Mehler, B., \& Reimer, B. (2017). Glass halffull: On-road glance metrics differentiate crashes from near-crashes in the 100-Car data. Accident Analysis and Prevention, 107(December 2016), 48-62. https://doi.org/10.1016/j.aap.2017.07.021

Shinar, D. (2017). Traffic safety and human behavior. Emerald Publishing Limited.

Shults, R. A., Elder, R. W., Sleet, D. A., Thompson, R. S., \& Nichols, J. L. (2004). Primary enforcement seat belt laws are effective even in the face of rising belt use rates. Accident Analysis and Prevention, 36(3), 491-493. https://doi.org/10.1016/S0001-4575(03)00038-1

Singh, S. (2015). Critical reasons for crashes investigated in the National Motor Vehicle Crash Causation Survey. National Highway Traffic Safety Administration, (February), 1-2.

Suykens, J. A. K., \& Vandewalle, J. (1999). Least Squares Support Vector Machine Classifiers. Neural Processing Letters, 9(3), 293-300. https://doi.org/10.1023/A:1018628609742

Tango, F., \& Botta, M. (2013). Real-time detection system of driver distraction using machine learning. IEEE Transactions on Intelligent Transportation Systems, 14(2), 894-905. https://doi.org/10.1109/TITS.2013.2247760

Vegega, M., Jones, B., \& Monk, C. (2013). Understanding the Effects of Distracted Driving and Developing Strategies to Reduce Resulting Deaths and Injuries: A Report to Congress, (December).

Victor, T., Dozza, M., Bärgman, J., Boda, C.-N., Engström, J., Flannagan, C., ... Markkula, G. (2015). Analysis of naturalistic driving study data: Safer glances, driver inattention, and crash risk.

Werneke, J., \& Vollrath, M. (2012). What does the driver look at? the influence of intersection characteristics on attention allocation and driving behavior. Accident Analysis and Prevention, 45, 610-619. https://doi.org/10.1016/j.aap.2011.09.048

Wickens, C. D. (2002). Multiple resources and performance prediction. Theoretical Issues in Ergonomics Science, 3(2), 159-177. https://doi.org/10.1080/14639220210123806

Wickens, C. D. (2008). Multiple Resources and Mental Workload. Human Factors, 50(3), 449455. https://doi.org/10.1518/001872008X288394.

Wollmer, M., Blaschke, C., Schindl, T., Schuller, B., Farber, B., Mayer, S., \& Trefflich, B. (2011). Online Driver Distraction Detection Using Long Short-Term Memory. Intelligent Transportation Systems, IEEE Transactions On, 12(2), 574-582. https://doi.org/10.1109/TITS.2011.2119483

Yeo, M. V. M., Li, X., Shen, K., \& Wilder-Smith, E. P. V. (2009). Can SVM be used for automatic EEG detection of drowsiness during car driving? Safety Science, 47(1), 115-124. https://doi.org/10.1016/j.ssci.2008.01.007

Young, R. (2012). Cognitive Distraction While Driving: A Critical Review of Definitions and Prevalence in Crashes. SAE International Journal of Passenger Cars - Electronic and Electrical Systems, 5(1), 2012-01-0967. https://doi.org/10.4271/2012-01-0967 